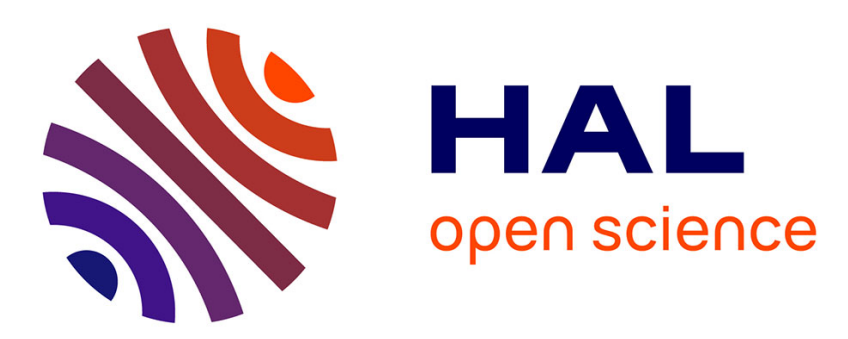

\title{
Dynamic Traffic Assignment for regional networks with traffic-dependent trip lengths and regional paths
}

Sergio Batista, Ludovic Leclercq, Monica Menendez

\section{To cite this version:}

Sergio Batista, Ludovic Leclercq, Monica Menendez. Dynamic Traffic Assignment for regional networks with traffic-dependent trip lengths and regional paths. Transportation research. Part C, Emerging technologies, 2021, 127, 26p. 10.1016/j.trc.2021.103076 . hal-03157510v2

\section{HAL Id: hal-03157510 \\ https://hal.science/hal-03157510v2}

Submitted on 26 Apr 2021

HAL is a multi-disciplinary open access archive for the deposit and dissemination of scientific research documents, whether they are published or not. The documents may come from teaching and research institutions in France or abroad, or from public or private research centers.
L'archive ouverte pluridisciplinaire HAL, est destinée au dépôt et à la diffusion de documents scientifiques de niveau recherche, publiés ou non, émanant des établissements d'enseignement et de recherche français ou étrangers, des laboratoires publics ou privés. 


\title{
Dynamic Traffic Assignment for regional networks with traffic-dependent trip lengths and regional paths
}

\author{
S. F. A. Batista ${ }^{\mathrm{a}, *}$, Ludovic Leclercq ${ }^{\mathrm{b}}$, Mónica Menéndez ${ }^{\mathrm{a}}$ \\ ${ }^{a}$ Division of Engineering, New York University Abu Dhabi, Saadiyat Marina District PO Box 129188 - Abu Dhabi, United Arab Emirates \\ ${ }^{b}$ Univ. Gustave Eiffel, Univ. Lyon, ENTPE, LICIT, Lyon, France
}

\begin{abstract}
The estimation of trip lengths has been proven to be a key feature for the application of aggregated traffic models based on the Macroscopic Fundamental Diagram. The paths and distances to be traveled by vehicles in regional networks vary over time, due to changes in the traffic conditions. In this paper, we develop a methodological framework to explicitly determine traffic-dependent regional paths and estimate their travel distances. This framework is incorporated into a dynamic traffic assignment module designed to target the Deterministic and Stochastic User Equilibrium in regional networks. We first discuss how regional paths and their characteristic trip lengths are influenced by changes in the regional traffic dynamics. We then test the proposed methodology for estimating traffic-dependent travel distances on small and medium-sized networks, considering a simulation environment. We show that our methodology provides good estimations of the traffic-dependent trip lengths. Our results also shed light on the importance of how time-dependent trip lengths influence the traffic dynamics in the regions.
\end{abstract}

Keywords: Traffic-dependent trip lengths, Regional paths, Regional Dynamic Traffic Assignment, Macroscopic Fundamental Diagram, Aggregated traffic models.

\section{Highlights}

- The distributions of trip lengths vary with traffic conditions.

- We develop a methodological framework for estimating explicit traffic-dependent trip length distributions and paths in the regional network.

- We discuss the integration of traffic-dependent trip lengths and paths into the traffic assignment for regional networks.

- We validate our methodology on small and medium-sized city networks, using an MFD simulation environment.

- The proposed methodology shows a good performance for estimating traffic-dependent trip lengths.

\section{Introduction}

Aggregated traffic models (Godfrey, 1969; Daganzo, 2007; Geroliminis and Daganzo, 2008; Vickrey, 2020) have been regarded as a powerful tool to describe the evolution of the traffic dynamics at the city network level, with a low computational effort. The application of these aggregated traffic models requires the partition of the city network (Figure 1 (a)) into regions where the traffic conditions are approximately homogeneous, i.e. vehicles travel at the same average speed. Several partitioning approaches have been discussed in the literature (Lopez et al., 2017; Saeedmanesh and Geroliminis, 2017; Ambühl et al., 2019). This step allows defining the regional network, where traffic

${ }^{*}$ Corresponding author. Tel. : +971 (0)2 6287698

Email address: sergio. batista@nyu.edu (S. F. A. Batista) 
(a)

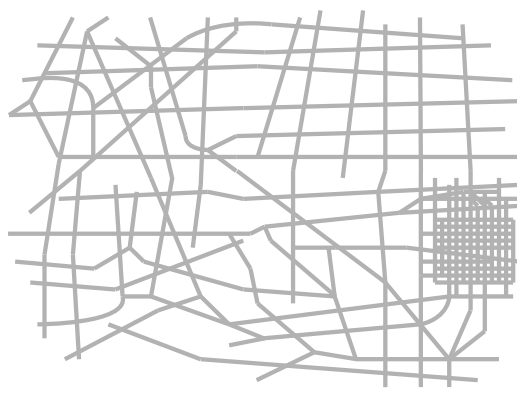

(b)

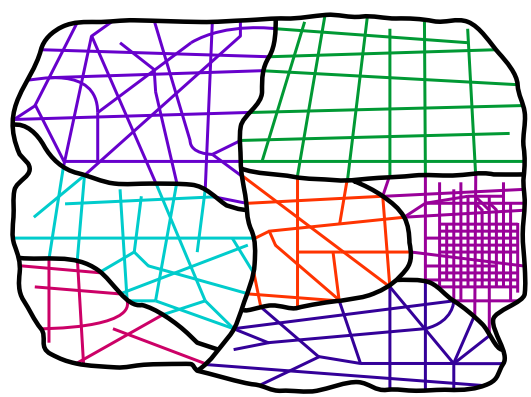

(c)

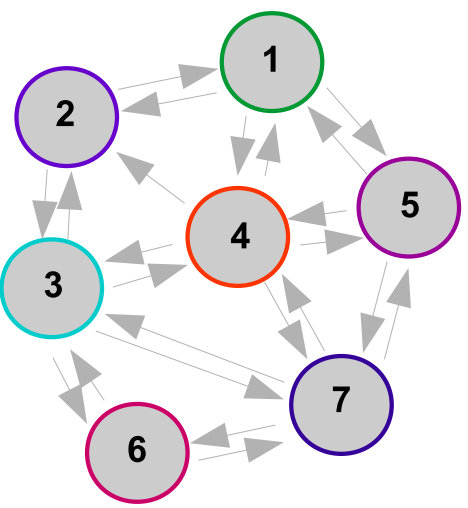

Figure 1: (a) City network. (b) Partitioning of the city network. (c) Graph representing the regional network.

is represented as exchange flows between regions and where routing options can be defined. We define $X$ as the set of all regions included in the regional network. Figure 1 (c) depicts the graph of the regional network equivalent to the partitioning defined in Figure 1 (b). The nodes represent the regions, and the connections between the regions are given by the allowed travel directions in the city network. The Macroscopic Fundamental Diagram (MFD) reflects the traffic conditions in each region $r \in X$. The MFD captures the relationship between the average circulating flow of vehicles $q_{r}([\mathrm{veh} / \mathrm{s}])$ and the accumulation $n_{r}$ ([veh]) in a generic region $r$. The evolution of the vehicles' accumulation $n_{r}$ in a region $\forall r \in X$ depends on the balance between their inflow $Q_{i n, r}(t)$ and outflow $Q_{\text {out }, r}(t)$ at a given small time interval $t$ :

$$
\frac{d n_{r}(t)}{d t}=Q_{i n, r}(t)-Q_{o u t, r}(t), t>0 \wedge \forall r \in X
$$

In the literature one can distinguish between two kinds of MFD traffic models: the accumulation-based model (Daganzo, 2007; Geroliminis and Daganzo, 2008; Mariotte and Leclercq, 2019); and the trip-based model (Vickrey, 2020; Arnott, 2013; Lamotte and Geroliminis, 2016; Mariotte et al., 2017; Leclercq et al., 2017; Mariotte and Leclercq, 2019; Jin, 2020). In this paper, we focus our attention only on the accumulation-based model.

Scaling-up a city into a regional network (see Figure 1 (a)-(c)) brings new challenges (Yildirimoglu and Geroliminis, 2014; Batista et al., 2019; Batista and Leclercq, 2019; Batista et al., in press.), mainly related to the definition and characterization of paths on the regional network. These represent the basis of any traffic assignment framework. Figure 2 (a) depicts four trips in the city network, that cross a different sequence of regions following the city network partitioning, i.e. they are associated with different paths in the regional network. Figure 2 (b) shows the corresponding paths in the regional network. A path in the regional network is defined as the ordered sequence of traveled regions from the Origin to the Destination $\left(\mathrm{OD}^{1}\right)$ regions (Batista et al., in press.). Figure 2 (b) shows the example of two regional paths crossing a different sequence of regions, and an internal path that represents trips that start and end within the same region. One can also observe that the different trips have different travel distances inside the regions they cross. This means that the regions defining a path are characterized by trip length distributions (see Figure 2 (c)); whereas trips in the city network are defined by a sequence of links with a fixed physical length. This key difference between paths in the city and regional networks has been recognized by Yildirimoglu and Geroliminis (2014) and Batista and Leclercq (2019) in designing route guidance frameworks for regional networks. The one discussed by Batista and Leclercq (2019) considers trip length distributions that are explicitly calculated, based on a set of trips in the city network and different levels of information from its partitioning. They assume the trip length distributions

\footnotetext{
${ }^{1}$ In this paper, we define od as the origin-destination pair in the city network. While OD stands for the Origin-Destination pair of regions in the regional network.
} 
(a)

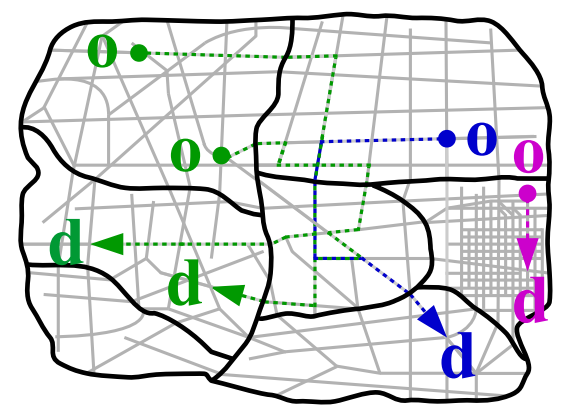

(b)

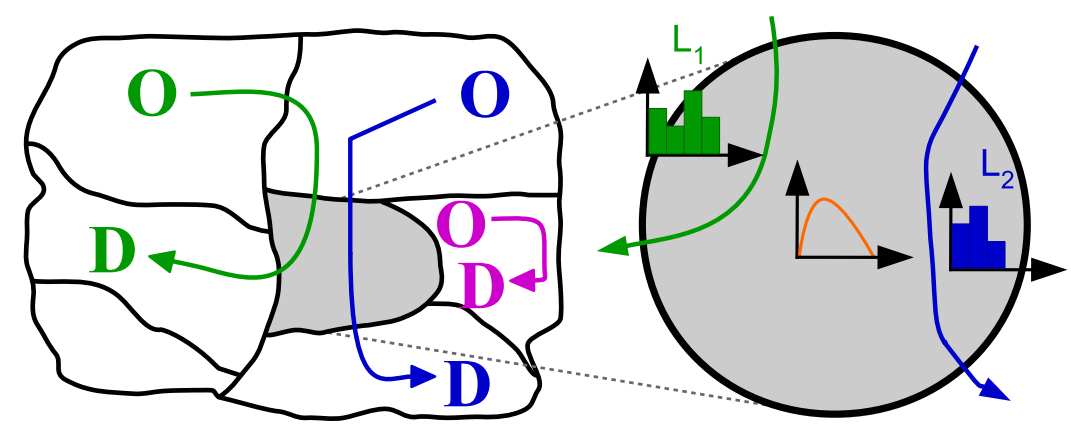

Figure 2: (a) Examples of trips in the city network. (b) The corresponding paths in the regional network, to the trips represented in (a). (c) Gray region that is crossed by two regional paths with trip length distributions $L_{1}$ and $L_{2}$.

to be static, i.e. not dependent on the traffic conditions. However, the travel distances of vehicles in the regions vary over time due to changes in the traffic conditions. For example, if the gray region (see Figure 2 (b)-(c)) becomes congested, drivers traveling on the dark blue trip might shift to the magenta one, crossing a different sequence of regions. This leads drivers to take detours in the city network, that are associated with other paths in the regional network. These detours change the trip length distributions. In this spirit, Yildirimoglu and Geroliminis (2014) proposed a route guidance framework based on the Multinomial Logit model where the trip lengths are traffic-dependent and implicitly calculated. Yildirimoglu et al. (2015) and Yildirimoglu et al. (2018) designed a control strategy coupled with route guidance, also with the trip lengths implicitly calculated. Ramezani et al. (2015) discussed a perimeter control strategy, where the trip lengths are calculated dynamically based on exchange flows between adjacent regions. However, most of the MFD-based control strategies and applications (Aboudolas and Geroliminis, 2013; Geroliminis et al., 2013; Kouvelas et al., 2017; Haddad, 2017; Jamshidnejad et al., 2017; Sirmatel and Geroliminis, 2017; Zhong et al., 2017; Yang et al., 2018; Sirmatel and Geroliminis, 2018; Mohajerpoor et al., 2019; Haitao et al., 2019; Yang et al., 2019; Ingole et al., 2020), assume the travel distance is identical for all drivers traveling on the same region. This assumption plays a major role in the traffic dynamics in the regions (Batista et al., 2019).

This paper sheds light on the importance of estimating traffic-dependent trip lengths for MFD-based applications. The main contributions of this paper are twofold. First, we propose a methodological framework for estimating trafficdependent distributions of trip lengths. Second, we discuss the extension of the Regional Dynamic Traffic Assignment (R-DTA) framework proposed by Batista and Leclercq (2019) to account for time-dependent trip lengths and paths on regional networks. However, we note that the proposed methodology for estimating traffic-dependent trip lengths can also be used on MFD-based control strategies and applications.

The remainder of this paper is organized as follows. In Sect. 2, we review the R-DTA and introduce the methodological framework for estimating traffic-dependent trip lengths. In Sect. 3, we investigate the time-dependence of trip lengths and test the proposed methodological framework on a static scenario as well as a simulation environment on a small city network. In Sect. 4, we also test the proposed methodological framework on a simulation environment and a medium-sized real city network. In Sect. 5, we outline the main conclusions of this paper and discuss future research directions.

\section{R-DTA and traffic-dependent paths and trip lengths on regional networks: methodological framework}

In Sect. 2.1, we briefly describe the methodology introduced by Batista et al. (2019) for estimating static (i.e. traffic independent) trip length distributions. In Sect. 2.2, we discuss a new methodological framework to estimate the traffic-dependent trip lengths. In Sect. 2.3, we discuss how the proposed methodology to estimate traffic-dependent trip lengths can be incorporated into the R-DTA framework proposed by Batista and Leclercq (2019).

In Table 1, we summarize the notation used in this paper. 
City and regional networks:

$o$

$d$

$O$

D

$p$

$r$

$a$

$l_{a}$

$X$

$R$

$N$

A

E

$\Gamma$

$K_{p}$

$\Omega^{O D}$

W

$N_{\text {od }}$

MFD models:

$t$

$q_{r}$

$n_{r}(t)$

$Q_{\text {in, } r}(t)$

$Q_{\text {out }, r}(t)$

$P_{r}\left(n_{r}\right)$

$v_{r}\left(n_{r}\right)$

$\bar{v}_{r}$

Trip lengths:

$L_{r p}$

$\bar{L}_{r p}$

$\hat{L}_{r p}$

$l_{r p}^{k}$
$\beta_{p}^{k}$

$\beta_{a r}$

$\omega_{r}\left(n_{r}\right)$

$\Delta h$

$v_{r}^{f f}$

S

$v^{*}$

$\alpha_{0}, \alpha_{i}$ and $\alpha_{i j}$

$\mu$
Origin node.

Destination node.

Origin region.

Destination region.

Regional path.

Generic region $r$ of the regional network.

Generic link of the city network.

Length of link a.

Set of regions that define the regional network.

Total number of regions in $X$.

Set of nodes that define the city network.

Set of links that define the city network.

Set of edges that define the regional network.

Set of virtual trips.

Number of virtual trips linked to a generic regional path $p$.

Regional choice set.

Set of regional OD pairs.

Total number of sampled origin-destination pairs in the city network.

Small time interval.

Average circulating flow of vehicles in region $r$.

Accumulation of vehicles in region $r$, during time interval $t$.

Inflow function of region $r$, during time interval $t$.

Outflow function of region $r$, during time interval $t$.

Production MFD.

Speed-MFD.

Mean speed in region $r$ over the time-period $\delta t$.

Set of trip lengths of regional path $p$ inside region $r$.

Average trip length of regional path $p$ inside region $r$.

Estimated trip length of generic region $r$ of regional path $p$.

Length of virtual trip $k$ that is associated with regional path $p$ and within region $r$.

Binary variable that equals 1 if virtual trip $k$ is associated with regional path $p$, or 0 otherwise.

Binary variable that equals 1 if link $a$ is in region $r$, or 0 otherwise.

Set of discretized speeds of region $r$.

Discretization amplitude of the speed-MFD for region $r$.

Free-flow speed of region $r$.

Total number of speed samples for region $r$.

Set of observed mean speeds.

Regression coefficients of the multi-linear model.

Number of common virtual trips in the $R$ sets. 
Table 1 - Continued from previous page

\begin{tabular}{ll}
\hline$U_{p}^{O D}$ & Utility function of regional path $p$ that connects the regional $O D$. \\
$T T_{p}^{O D}$ & Travel time of regional path $p$ that connects the regional $O D$. \\
$T$ & Simulation period. \\
$\delta t$ & $\begin{array}{l}\text { Duration of the sub-interval for calculating the network equilibrium for the quasi-static } \\
\text { approximation. }\end{array}$ \\
$N_{\max }$ & Total number of the MSA descent step iterations. \\
$M(\lambda)$ & $\begin{array}{l}\text { Number of violations that represent the difference of the regional path flows between } \\
\text { consecutive descent step iterations of the Method of Successive Averages. }\end{array}$ \\
$\Phi$ & Threshold that defines the tolerance for the number of violations. \\
$G a p$ & Relative gap between the regional paths travel time and the travel time at the network \\
$t o l$ & equilibrium. \\
$b$ & Tolerance for the Gap criterion. \\
$\zeta$ & MSA iteration. \\
$\Delta t$ & MSA descent step.
\end{tabular}

\subsection{Estimation of trip lengths distributions: a review}

Batista et al. (2019) recognized the importance of the calibration of trip lengths for predicting the traffic dynamics in the regions, using an aggregated MFD traffic model. The authors proposed a methodology to explicitly determine static (i.e. not accounting for changes in the traffic dynamics) distributions of trip lengths, that require the information of a set of trips in the city network as well as the city network partitioning. The partitioning can be done by applying any of the approaches discussed in the literature (Lopez et al., 2017; Saeedmanesh and Geroliminis, 2017; Ambühl et al., 2019), ensuring that the regions are connected and compact. The most challenging question is how to get a set of trips in the city network. One option is to use data-driven methods. However, in the best scenario, one can only get a partial set of the daily trip patterns in the city network. Thus, we need to ensure that this partial set is representative of the full daily trip patterns in the city. How to ensure such representativeness, although important, is out of the scope of this study. For instance, Batista et al. (2019) proposed to construct a set of virtual trips by randomly sampling multiple origin-destination (od) pairs in the city network and then calculating the shortest-trips in distance for each of them. Let $\Gamma$ be this set of static virtual trips. The set of virtual trips requires a complex calibration task as discussed by Batista et al. (2019). The authors are also currently working on a more intelligent sampling procedure that can identify a representative subset of od pairs from all possible trips connecting one regional OD pair (Batista et al., 2021), which also considers the spatial correlations between the nodes of the city network. This will drastically reduce the computational burden for determining the set of virtual trips. In any case, the paths on the regional network are determined by scaling-up these trips following the sequence of regions they cross, and according to the city network partitioning. For each regional OD pair, the paths are ranked according to their level of significance. The level of significance of a path $p$ on the regional network is related to the total number of trips, $K_{p}$, it has associated. For example, the most significant path connecting one generic OD pair is the one with the largest $K_{p}$. We define the regional choice set as $\Omega^{O D}, \forall(O, D) \in W$, where $W$ is the set of all regional OD pairs, to contain the most significant paths on the regional network for each OD pair.

Using the methodology from Batista et al. (2019), we can determine the distributions of trip lengths, $L_{r p}$, for region $r$ and path $p$. We refer to $L_{r p}$ as the reference distribution of trip lengths. They are calculated based on $\Gamma$, and different levels of information about the sequence of regions crossed by these trips. The latter ranges from no prior information about the previous and next regions to be traveled by the trips, to their path on the regional network. In this paper, we focus on the most detailed level of information, i.e. the related path on the regional network, following the discussion in Batista et al. (2019). The reference distribution of trip lengths $L_{r p}$ is then:

$$
L_{r p}=\left\{\bigcup_{\forall \in \in \Gamma} \beta_{p}^{k} l_{r p}^{k}\right\}
$$


where $l_{r}^{k}$ is the length of virtual trip $k$, associated with path $p$ and inside region $r$; and $\beta_{p}^{k}$ is a binary variable that equals 1 if virtual trip $k$ is associated with path $p$, or 0 otherwise.

The average trip length $\bar{L}_{r p}$ of a path $p$ in region $r$ is then:

$$
\bar{L}_{r p}=\frac{\sum_{k} \beta_{p}^{k} l_{r p}^{k}}{\sum_{k} \beta_{p}^{k}}, \forall k \in \Gamma
$$

\subsection{Estimation of traffic-dependent trip lengths}

Yildirimoglu and Geroliminis (2014) and Batista et al. (2019) recognize that the travel distances are influenced by the traffic dynamics in the regions. Generally, this means that the distribution of trip lengths $L_{r p}$ depends on the traffic conditions in all regions $r \in X$, that are regulated by their speed-MFDs $v_{m}\left(n_{m}\right)$ as:

$$
L_{r p}=f\left(v_{1}\left(n_{1}\right), \ldots, v_{m}\left(n_{m}\right)\right), \forall m \in X
$$

where $v_{m}\left(n_{m}\right)$ is the speed-MFD that reflects the traffic conditions inside a generic region $m \in X$ and $n_{m}$ is the vehicles' accumulation for that region. For a generic path $p, L_{r p}$ is a multi-dimensional function where the number of dimensions depends on the total number of regions $R$ that define $X$.

The question is then how to determine the distribution of trip lengths $L_{r p}$ according to the changes in the traffic conditions, without the need to recalculate the set of time-dependent virtual trips online during the MFD simulation. The computational cost of doing so largely increases with the number of trips and size of the city network. In this section, we propose an alternative formulation to do an online estimation of the distribution of trip lengths $L_{r p}$, that consists of three steps as depicted in Figure 3. The first step consists of discretizing the multi-dimensional space, considering the speed-MFDs of all regions, to construct the numerical grid. In the second step, we determine the set of time-dependent virtual trips for each regional OD pair and each point in the numerical grid. This permits constructing a library of time-dependent distributions of trip lengths $L_{r p}$ for each point of the numerical grid. These first two steps are done offline. The third step consists of estimating the distributions of trip lengths $\hat{L}_{r p}$ for a given set of observed mean speeds $v^{*}$ in the regions. This step is done online during the MFD simulation and utilizes the library of time-dependent distributions of trip lengths $L_{r p}$. Below, we describe these three steps in more detail.

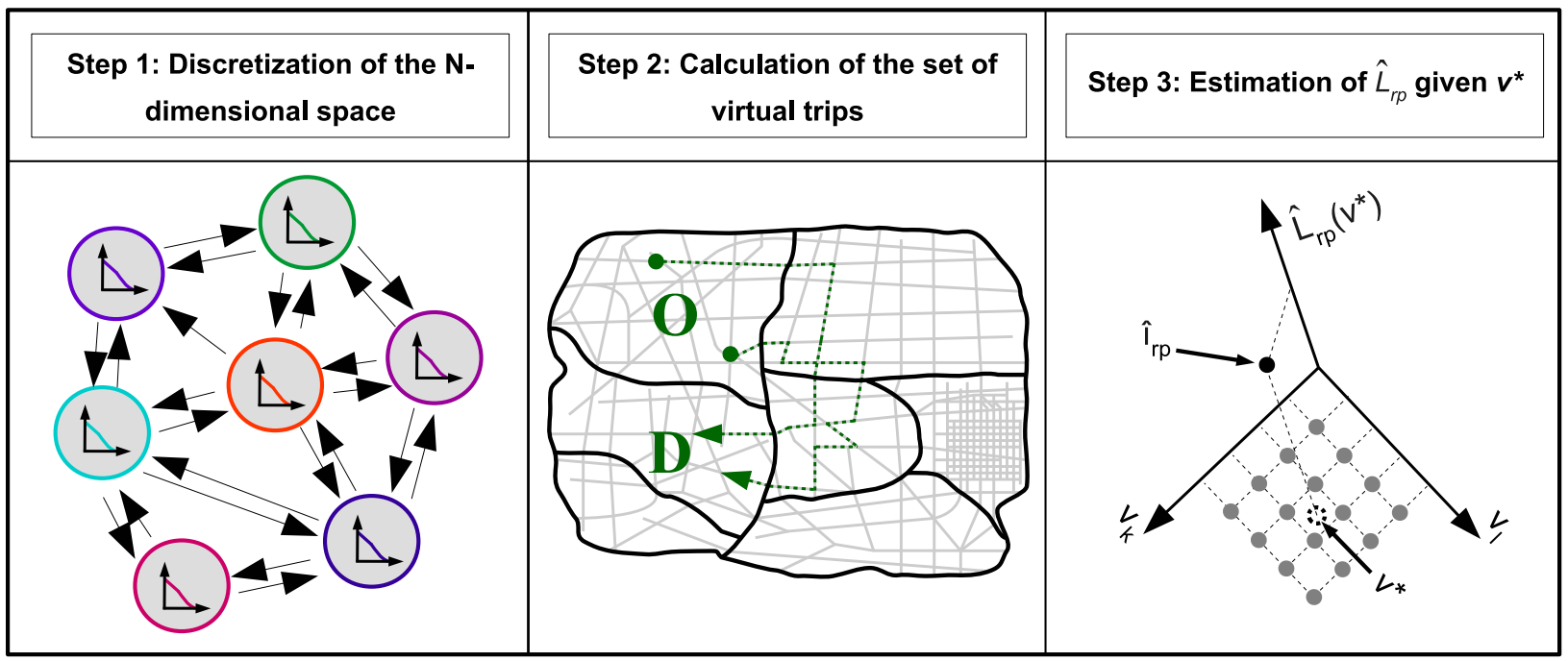

Figure 3: (a) Step 1: Set of speed-MFDs of the regional network that represents the multi-dimensional space (see Eq. 4). (b) Step 2: Calculation of the sets of traffic-dependent shortest-trips for each point in the numerical grid. (c) Step 3: Estimation of the trip length sample $\hat{L}_{r p}$ for a given a set of observed speeds $v^{*}$. 


\subsubsection{Discretization of the $\mathrm{N}$-dimensional space}

The first step defines a multi-dimensional grid. As noted in Eq. 4, the distribution of trip lengths $L_{r p}$ depends on the observed traffic conditions on all regions of the network, which are regulated by the speed-MFD (i.e. the relationship between the mean speed $\bar{v}_{r}$ and the accumulation $n_{r}$ in each region). Figure 3 (a) depicts an example of a regional network, where the speed-MFDs characterize all possible traffic states in each region, i.e. the range of possible observed mean speeds in each region. Following this rationale, we build the multi-dimensional grid by discretizing the speed-MFD into intervals of equal amplitude $\Delta h=\frac{v_{r}^{f f}}{S}$, where $v_{r}^{f f}$ is the free-flow speed of region $r$, and $S$ is the total number of discretized points of the speed-MFD. Notice that different parts of the speed-MFD can be discretized according to different criteria (i.e. values of $S$ ). We define $\omega_{r}\left(n_{r}\right), \forall r \in X$ as the set of discretized speeds of a generic region $r$. This permits constructing a uniform multi-dimensional grid of points, for which one has to determine the set of traffic-dependent virtual trips. This step is done offline, which means that we do not perform any MFD simulation at this stage. Instead, each point in the multi-dimensional grid represents one out of many possible observed congestion states of the entire traffic network, as permitted by the speed-MFDs of all regions.

\subsubsection{Calculation of the set of shortest virtual trips in time}

The second step calculates a set of time-dependent virtual trips for each regional OD pair and each point of the multi-dimensional numerical grid. Initially, for each OD pair, we uniformly sample a set of $N_{o d}$ pairs of nodes in the city network (Batista et al., 2019, in press.). These sets are considered the same for all points of the numerical grid. Then, for each point of the numerical grid and each OD pair, we calculate the set of time-dependent virtual trips (see Figure 3 (b)) based on the fixed set of od pairs. We consider the calculation of shortest-trips in time, where the travel time of a generic link $a$ is determined as:

$$
t_{a}=\frac{l_{a} \beta_{a r}}{v_{r}^{j}\left(n_{r}\right)}, \forall a \in A \wedge \forall j=1, \ldots, S
$$

where $l_{a}$ is the length of link $a ; A$ is the full set of links that define the city network; and $\beta_{a r}$ is a binary variable that equals 1 if link $a$ is in region $r$, or 0 otherwise.

Each set of time-dependent virtual trips determined for each point of the numerical grid, is then scaled-up according to the sequence of traveled regions, i.e. according to the definition of the city network partitioning. This permits determining the corresponding paths in the regional network, and explicitly characterizes their distributions of trip lengths $L_{r p}$.

\subsubsection{Estimation of $\hat{L}_{r p}$ given $v^{*}$}

The third step is the online estimation of the distributions of trip lengths $\hat{L}_{r p}$ for a given set of observed mean speeds in the regions $v^{*}=\left\{v_{r}^{*}\right\}, \forall r \in X$. Figure 3 (c) shows a schematic representation of this step. For ease of the illustrative scheme, we show an example of a two-regions network, where $v_{k}$ and $v_{l}$ are the respective speed-MFDs. The goal is to estimate the samples $\hat{l}_{r p}$ of the distribution of travel distances $\hat{L}_{r p}$, given a set of observed speeds $v^{*}$. For this step, we make the following important assumption:

Assumption. We assume that locally in the vicinity of $v^{*}$, the topology of the $L_{r p}$ function, defined in Eq. 4 , can be approximated by a hyper-plane in the R-dimensional space.

We discuss the coherence of this assumption in the Sect. 3.3. Note that, a hyper-plane is spanned by $R-1$ linearly independent vectors. For this reason and each OD pair, we select the $R$ points in the vicinity of $v^{*}$, for which we gather the distributions of trip lengths $L_{r p}$ from the library. We then identify the total number $\mu$ of common trips to all $R$ sets of virtual trips. Let $l_{r p}$ be a generic trip length sample from the distribution $L_{r p}$, of each of the common trips. The goal is to estimate the samples $\hat{l}_{r p}$ that define the distribution of trip lengths $\hat{L}_{r p}$. For this purpose, we fit a multi-dimensional linear regression model to the $R$ samples $l_{r p}$, and determine the predictors $\alpha_{0}, \alpha_{i}$ and $\alpha_{i j}$, as follows:

$$
l_{r p}=\alpha_{0}+\sum_{i \in X}\left(\alpha_{i} v_{i}+\sum_{\substack{j \in X \\ i \neq j}} \alpha_{i j} v_{i} v_{j}\right), \forall r \in p \wedge \forall p \in \Omega^{O D}
$$


where $v_{i}$ are the speed samples of region $i$, i.e. the coordinates in the numerical grid. Note that, we only account for the paths in the regional network, that are tagged as available in the previous step.

In the next step, we estimate each sample $\hat{l}_{r p}$ as follows:

$$
\hat{l}_{r p}=\alpha_{0}+\sum_{i \in X}\left(\alpha_{i} v_{i}^{*}+\sum_{\substack{j \in X \\ i \neq j}} \alpha_{i j} v_{i}^{*} v_{j}^{*}\right), \forall r \in p \wedge \forall p \in \Omega^{O D}
$$

This methodology permits to determine the samples $\hat{l}_{r p}$ of trip lengths that define the estimated distribution $\hat{L}_{r p}=$ $\left\{\hat{l}_{r p}\right\}, \forall r \in p \wedge \forall p \in \Omega^{O D}$. Note that, this distribution only encompasses samples $\hat{l}_{r p}$ of the identified common trips to all $R$ sets of virtual trips that are gathered from the library.

This methodology can be implemented to estimate traffic-dependent trip lengths on all MFD-based applications, where one needs to update the trip lengths according to the traffic conditions in the regions. Important examples include applications of MFD-based models for perimeter control and the R-DTA described by Batista and Leclercq (2019). In the next section, we discuss how the developed methodological framework to estimate traffic-dependent trip lengths can be incorporated into the R-DTA.

\subsection{Regional Dynamic Traffic Assignment with time-dependent trip lengths}

In this section, we discuss how to determine the classical Deterministic and Stochastic User Equilibrium in regional networks (Batista and Leclercq, 2019), considering traffic-dependent regional paths and trip lengths. We consider the R-DTA framework proposed by Batista and Leclercq (2019). Figure 4 depicts a schematic representation of the different steps of the R-DTA. For notation purposes, we define the city network as the connected graph $G(N, A)$, where $N$ and $A$ represent the set of nodes and links. Similarly, the regional network corresponds to the connected graph $G(X, E)$, where $E$ defines the edges that connect the regions (see Figure 1 (c)).

In this paper, we determine the regional network equilibrium using a quasi-static approximation. This means that the total simulation period $T$ is divided into several sub-intervals of duration $\delta t$, where the network equilibrium is calculated. A schematic representation of this procedure is depicted in Figure 4. The regional path flows are kept constant over each $\delta t$. The length of $\delta t$ can be adjusted in different trials for better capturing rapid changes in the demand or sudden changes in the regional traffic dynamics.

In the R-DTA version proposed by Batista and Leclercq (2019), the set of regional paths and trip length distributions are determined following the procedure discussed in Sect. 2.1 and uploaded only at the beginning of the simulation time, i.e. at $t=0$. Both of them are obtained from a static set of virtual trips that do not account for the detour ratios due to the changes in the regional traffic dynamics. The box within the red dashed line in Figure 4 represents the inner-loop where the network equilibrium is calculated at each interval $\delta t$, until the total simulation time $T$ is reached. In this paper, we also target the Deterministic and Stochastic User Equilibrium principles as in Batista and Leclercq (2019). The authors have noted that the travel time of a regional path, $T T_{p}^{O D}, \forall p \in \Omega^{O D} \wedge \forall(O, D) \in W$, depends on the empirical distributions of trip lengths $\left\{L_{r p}\right\}$ and on the time-varying speed MFD $v_{r}\left(n_{r}\right)$. It is determined as:

$$
T T_{p}^{O D}=\sum_{r \in X}\left(\frac{L_{r p}}{v_{r}\left(n_{r}\right)}\right) \beta_{r p}, \forall p \in \Omega^{O D} \wedge \forall(O, D) \in W
$$

Note that, in Eq. 8, the trip length distributions $L_{r p}$ can be directly and explicitly determined based on a set of traffic-dependent virtual trips, or estimated using the methodological framework described in the previous Sect. 2.2.

Batista and Leclercq (2019) approximate the utility function of regional path $p, U_{p}^{O D}$, by doing a first order Taylor series expansion of Eq. 8 around the mean values $\bar{L}_{r p}$ and $\bar{v}_{r}$. The mathematical details of these calculations are described in Batista and Leclercq (2019). In the case of the Deterministic User Equilibrium (DUE), none of the terms are considered to be distributed, and $U_{p}^{O D}$ is calculated as:

$$
U_{p}^{O D}=\sum_{r \in X}\left(\frac{\bar{L}_{r p}}{\bar{v}_{r}}\right) \beta_{r p}, \forall p \in \Omega^{O D} \wedge \forall(O, D) \in W
$$



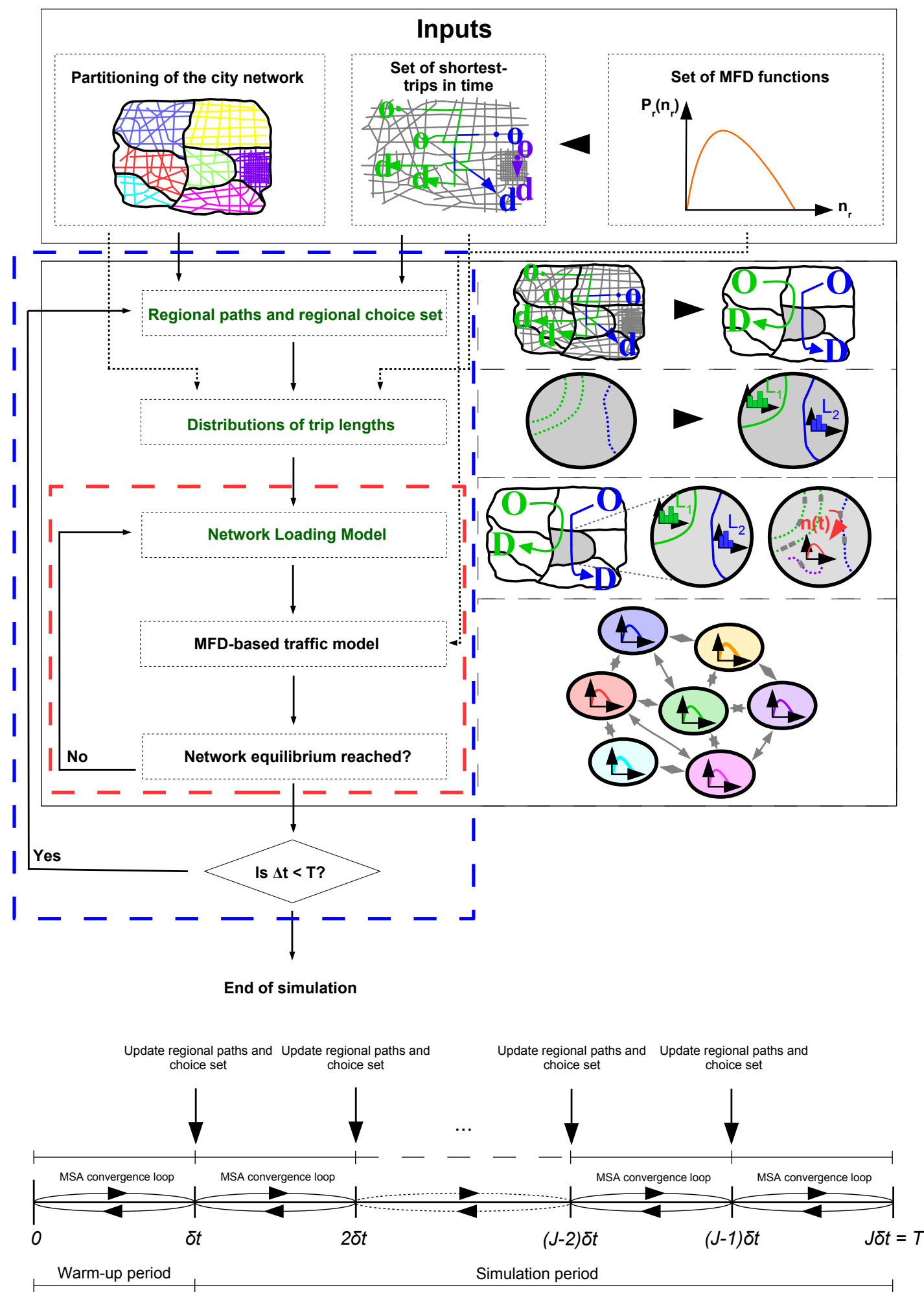

Figure 4: Flowchart of the R-DTA that accounts for time-dependent trip lengths and regional paths. A scheme explaining the quasi-static approximation to calculate the dynamic regional network equilibrium is also depicted. 
In the case of the Stochastic User Equilibrium (SUE), both terms are considered to be distributed, and the first order Taylor series expansion yields that $U_{p}^{O D}$ is:

$$
U_{p}^{O D}=\sum_{r \in X}\left(\frac{\bar{L}_{r p}}{\bar{v}_{r}}+\frac{L_{r p}}{\bar{v}_{r}}-\frac{\bar{L}_{r p} v_{r}}{\bar{v}_{r}^{2}}\right) \beta_{r p}, \forall p \in \Omega^{O D} \wedge \forall(O, D) \in W
$$

Both of the regional network equilibria are calculated using the classical Method of Successive Averages (MSA). At each descent step $b$, in the case of the DUE, drivers are assigned to the regional path with minimal perceived utility (see Eq. 9), based on an all-or-nothing procedure. In the case of the SUE, Monte Carlo simulations are used to account for the distributions $\left\{L_{r p}\right\}$ and $v_{r}\left(n_{r}\right)$ in Eq. 10. The goal is to draw samples from these two distributions and locally solve deterministic problems. This means that for each set of draws, drivers are also assigned based on an all-or-nothing procedure to the path with the minimal perceived utility. The new regional path flows are updated by averaging over the choices of drivers for all draws. The regional network equilibrium is achieved when the relative Gap (Sbayti et al., 2007) is inferior to a pre-defined tolerance and/or when the number of violations $M(\lambda)$ (Sbayti et al., 2007 ) is inferior to a given threshold $\Phi$. The relative Gap is an indicator that describes how far are the path travel times from the User Equilibrium conditions (Sbayti et al., 2007). The number of violations represents the difference in the regional path flows between two consecutive descent step iterations of the Method of Successive Averages. There is also a maximum number $N_{\max }$ of allowed iterations. To ensure the good convergence properties of the MSA, we need to choose an adequate step size of $e t a=\frac{1}{s}$.

In this paper, we propose to extend this R-DTA framework to include an outer loop represented by the blue dashed line in Figure 4. The goal is to update the set of regional paths and trip lengths for the next assignment period $\delta t$, based on the observed traffic conditions from the previous period, i.e. $v^{*}$. This proposed extension works as follows. The first period $\delta t$ represents a warm-up period that corresponds to the network loading. We need to determine the available regional paths and their trip lengths. To do so, we look for the $R$ closest points in the vicinity of $v^{*}$, and we gather the trip lengths sets from the library as discussed in the previous section. We tag the common regional paths to all $R$ sets as available in $\Omega^{O D}$. All the remaining paths are set as unavailable for the drivers' choices. The trip lengths are estimated using the methodology discussed in the previous section. We then load the regional paths and determine the regional network equilibrium that corresponds to the inner-loop. For the next period between $\delta t$ and $2 \delta t$, we update the regional paths tags and their trip lengths in the outer-loop, according to the observed traffic conditions $\left(v^{*}=\left\{v_{r}^{*}\right\}, \forall r \in X\right)$ of the previous period. We then determine again the network equilibrium in the inner-loop. This process of the inner and outer loops is repeated at every $\delta t$, over the cumulative period $\Delta t(g)=\Delta t(g-1)+\delta t<T$, where $g$ is an iterative variable over the different assignment periods. The maximum value of $h$ is reached at $T / \delta t$. Note that, in Figure 4, the first $\delta t$ is depicted as the warm-up period only for illustration purposes. This can be adjusted in different trials.

\section{Analysis of the time-dependent trip lengths on a small city network}

We now discuss the implementation of the methodological framework introduced in the previous section. First, we investigate how the traffic dynamics influence the significance level of paths and trip lengths. Second, we discuss how to properly calibrate the number of od pairs, $N_{o d}$, as well as of the number of points in the numerical grid. Third, we investigate how the proposed methodological framework performs in an MFD simulation environment. These tests are performed on a small city network.

\subsection{Description of the test network and demand scenarios}

The small size city network is the $6^{\text {th }}$ district of Lyon (France) depicted in Fig. 5 (a). We refer to this network as L6. It contains 757 links and 431 nodes, and it is partitioned into four regions, for which we fitted the speed-MFD functions depicted in Fig. 5 (b). The free-flow speeds are $v_{r}^{f f}=\{4.95,4.33,4.87,5.14\}, \forall r=1, \ldots, 4$, while the critical speeds are $v_{r}^{\text {crit. }}=\{2.8,3.2,3.1,2.9\}, \forall r=1, \ldots, 4$. We note that in this section, the speeds are expressed in $\mathrm{m} / \mathrm{s}$. We have set up the free-flow speeds to around $5 \mathrm{~m} / \mathrm{s}$ (i.e. $20 \mathrm{~km} / \mathrm{h}$ ) as this network represents a residential neighborhood composed of mainly one-way streets with a lot of parking and crossing pedestrians. The definition of the network partitioning is based on administrative regions as defined by the municipality, and as also discussed in 
Batista et al. (2019) and Batista and Leclercq (2019). The speed-MFDs are calibrated using a similar procedure as the one presented in these two studies.
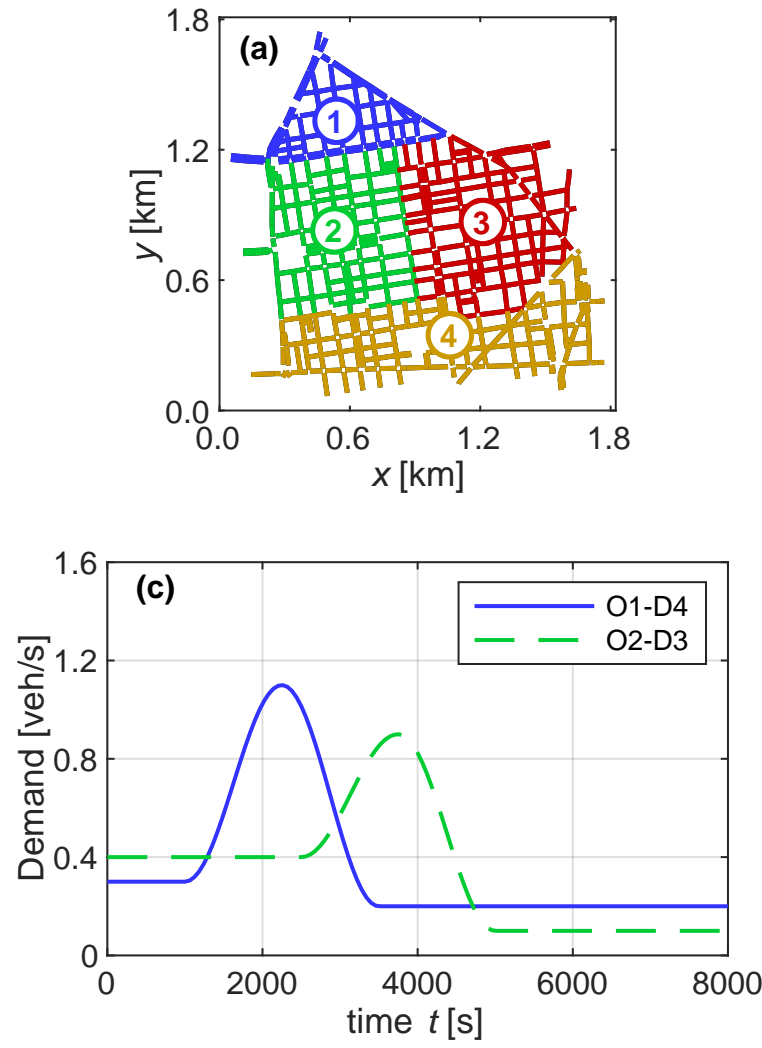
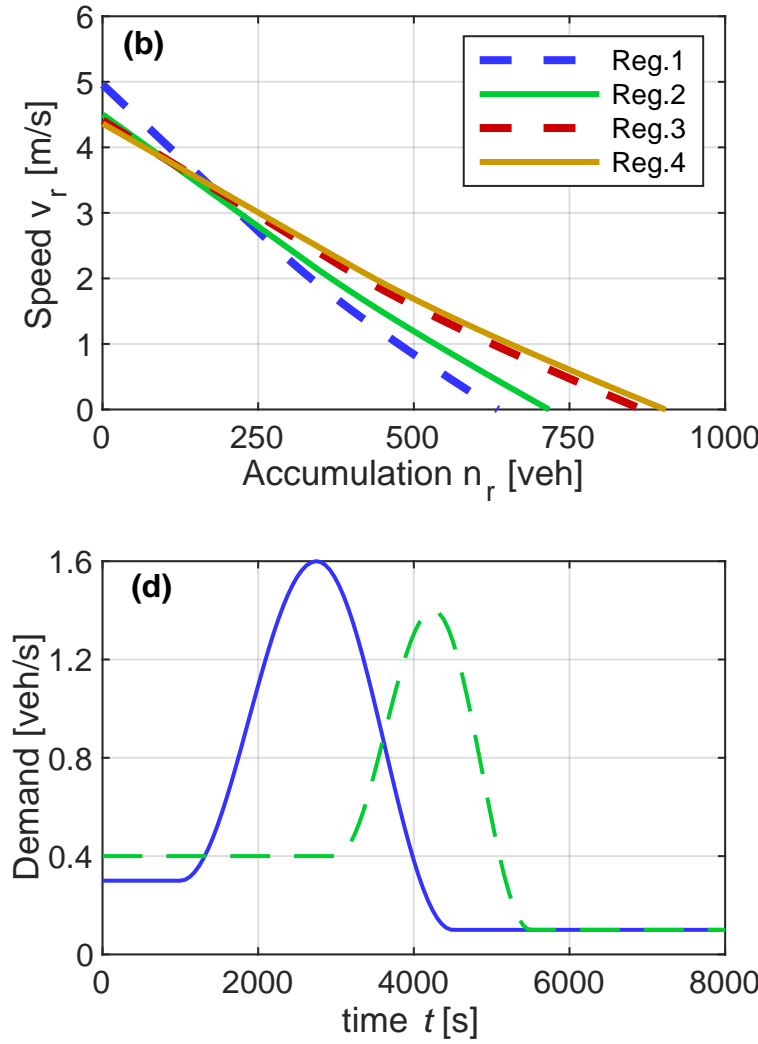

Figure 5: (a) $6^{\text {th }}$ district Lyon network (L6) divided into four regions. (b) Speed-MFDs of the L6 network. The demand scenarios for the L6 network are depicted in (c) for a free-flow case and in (d) for a congested case.

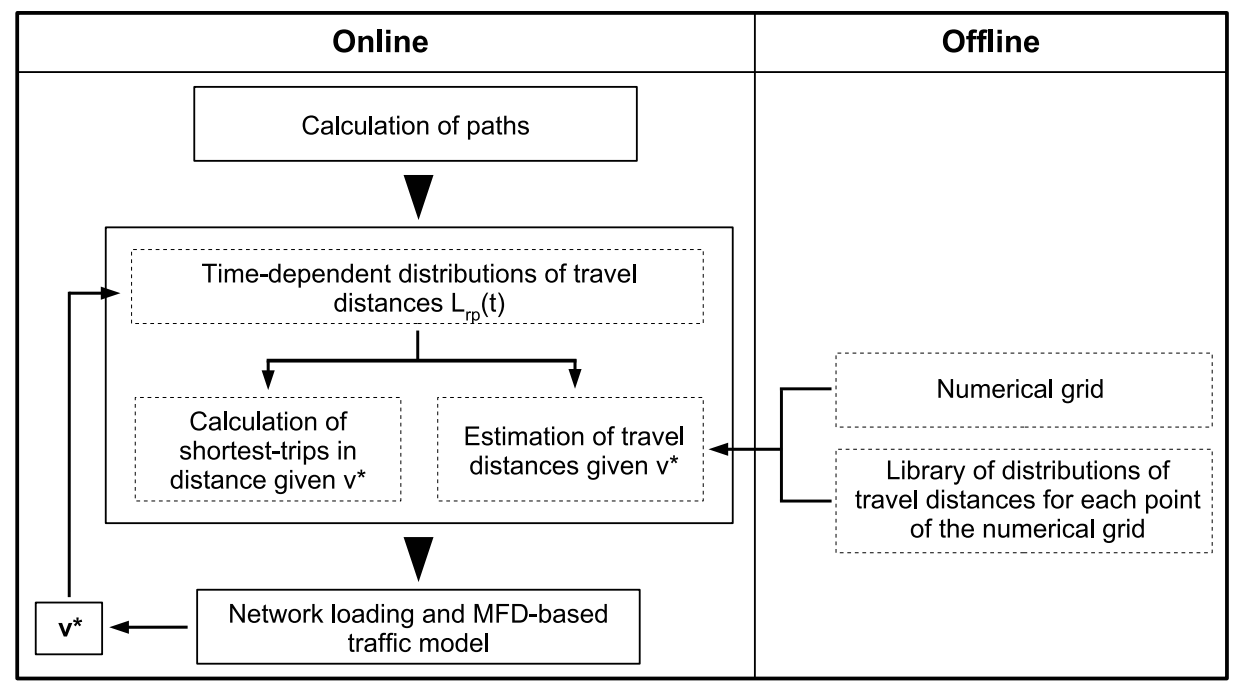

Figure 6: Flowchart representing the online validation, i.e. in an MFD simulation environment, of the developed methodological framework for estimating the trip length distributions. 
The testing of the methodology to estimate the time-dependent trip lengths described in the previous section includes two steps. First, we investigate how the traffic conditions influence the significance level $K_{p}$ and trip lengths of both an internal and regional path in the L6 network. We focus on paths $p=\{1\}$ and $p=\{124\}$ (Fig. 5 (a)). We then estimate the trip lengths for these two paths for different settings of the traffic conditions in the regions. For this test, we construct the multi-dimensional numerical grid by discretizing the speed-MFDs of the four regions as $\omega_{1}\left(n_{1}\right) \in[0.15: \Delta h: 4.95], \omega_{2}\left(n_{2}\right) \in[0.33: \Delta h: 4.33], \omega_{3}\left(n_{3}\right) \in[0.07: \Delta h: 4.87]$ and $\omega_{4}\left(n_{4}\right) \in[0.34: \delta v: 5.14]$, where $\Delta h=0.4(\mathrm{~m} / \mathrm{s})$. For each point in the multi-dimensional grid, we sample $N_{o d}=200$ od pairs in the Origin and Destination regions of the paths.

Second, we investigate how the methodology to estimate the trip lengths performs in an MFD simulation environment on the L6. To that end, we discuss how to properly calibrate $N_{o d}$ for the set of trips and the number of points $S$ for discretizing the speed-MFDs to construct the multi-dimensional numerical grid. We then perform the MFDsimulations considering the proper calibration of $N_{o d}$ and $S$. With this, we can validate the developed methodology to estimate online (i.e. in the MFD simulation environment) the traffic-dependent distributions of trip lengths. Fig. 6 depicts a flowchart that shows how this validation process is done. This consists of two approaches. One of these approaches represents the benchmark where the distributions of trip lengths are explicitly determined based on the direct recalculation of the time-dependent set of virtual trips given a set of observed mean speeds $v^{*}$ in the regions. In the other approach, we utilize the developed methodology to estimate the distributions of trip lengths for a given observed set of mean speeds $v^{*}$. This methodology takes as an input the definition of the multi-dimensional grid and the library of distributions of trip lengths $L_{r p}$ calculated for each OD pair and each point of the grid. These two inputs are calculated offline as depicted in Fig. 6.

For the simulations, we set a simulation period of $T=8000$ seconds, that is split into several intervals of length $\delta t=200$ seconds. For each interval, we determine the DUE. The equilibrium conditions are achieved when Gap $\leq 0.2$ and when $M(\lambda) \leq 0$. We also set a maximum number of descent step iterations $N_{\max }=100$. We set two demand scenarios: (i) a demand scenario where all regions are in free-flow conditions (Fig. 5 (c)); and (ii) another one where regions 2 and 3 become congested (Fig. 5 (d)). The demand scenarios are composed by two OD pairs: 14; and 2-3. We have in total seven possible regional paths. Based on a full enumeration, the regional choice sets are $\Omega^{14}=\{124,134,1234,1324\}$ and $\Omega^{23}=\{23,243,213\}$. The traffic dynamics are reproduced using an accumulationbased MFD-model (Daganzo, 2007; Mariotte and Leclercq, 2019).

\subsection{Time-dependence of the trip lengths on the traffic states}

In this section, we investigate how the traffic conditions influence the significance level $K_{p}$ and the average trip lengths $\bar{L}_{r p}$ of the paths $p=\{1\}$ and $p=\{124\}$ of the L6 network. The first $p=\{1\}$ represents an internal path within region 1, i.e. for trips that happen only inside this region. The second $p=\{124\}$ represents a regional path that permits to travel from regions 1 to 4 . Fig. 7 depicts the evolution of the significance level $K_{p}$ and the average trip lengths $\bar{L}_{r p}$ of $p=\{1\}$ and $p=\{124\}$, as a function of the traffic conditions. For $p=\{1\}$, we fix three values of $\bar{v}_{1}=4.95,2.8,1.6$ $(\mathrm{m} / \mathrm{s})$ and vary $\bar{v}_{2}$ and $\bar{v}_{3}$. Region 4 is maintained at the free-flow speed. For the regional path $p=\{124\}$ we maintain regions 1 and 4 at the free-flow speed and vary $\bar{v}_{2}$ and $\bar{v}_{3}$.

We first focus our analysis on the internal path $p=\{1\}$. We observe that under free-flow conditions in region 1 , the influence of the traffic dynamics of regions 2 and 3, on $K_{p}$ and the average trip length $\bar{L}_{1}$, are negligible. This is evidenced by the negligible difference of 2 trips in Fig. 7 (a) and of 1 meter in Fig. 7 (b). However, the influence of the traffic dynamics of regions 2 and 3 on $K_{p}$ and $\bar{L}_{1}$, become more important as $\bar{v}_{1}$ decreases. As region 1 becomes more congested while regions 2 and/or 3 are still in free-flow conditions, $K_{p}$ decreases, and therefore $\bar{L}_{1}$ decreases as well. A lower $\bar{v}_{1}$, means longer link travel times in region 1. This leads to detours in the virtual trips, as regions 2 and 3 present shorter link travel times than region 1 . As region 1 becomes more congested, a driver that would travel on the internal path $p=\{1\}$, will now shift to other paths, such as $p=\{1,2,1\}, p=\{1,3,1\}, p=\{1,3,2,1\}$ or $p=\{1,2,3,1\}$. This causes a decrease of $K_{p}$ and consequently of $\bar{L}_{1}$, as evidenced by the blue color in Fig. 7 (e) and 7 (f). However, as regions 2 and 3 become also more congested, i.e. $\bar{v}_{2}$ and $\bar{v}_{3}$ decrease, the link travel times of these regions increase, leading drivers to shift back again to internal trips in region 1 . This causes the increase of $K_{p}$ and consequently of $\bar{L}_{1}$, as evidenced by the yellow color in Fig. 7 (e) and 7 (f).

We can also observe a similar trend for $p=\{124\}$. When region 2 is in free-flow conditions, there are no significant changes in $K_{p}$ and the average trip lengths. However, as region 2 becomes congested and region 3 is in 

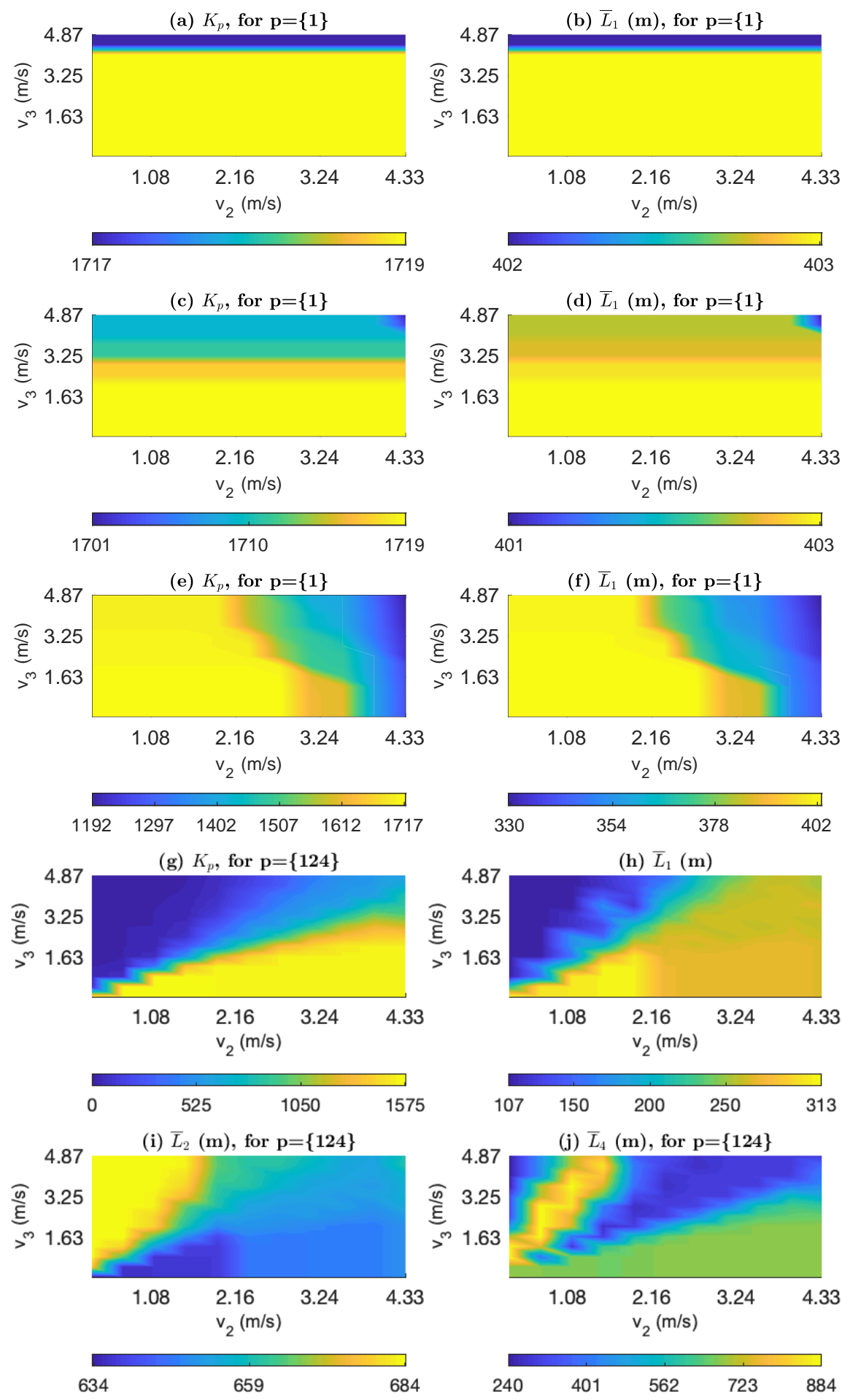

Figure 7: (a - f) Evolution of the significance level $K_{p}$ and average trip lengths $\bar{L}_{1}$ for internal path $p=\{1\}$. The results are depicted for $\bar{v}_{1}=4.95 \mathrm{~m} / \mathrm{s}$ (a-b), $\bar{v}_{1}=2.8 \mathrm{~m} / \mathrm{s}$ (c-d) and for $\bar{v}_{1}=1.6 \mathrm{~m} / \mathrm{s}$ (e-f). (g) Evolution of the significance level $K_{p}$ for regional path $p=\{124\}$ as a function of the traffic dynamics in regions 2 and 3. Evolution of the average trip lengths for the Origin (h), Intermediate (i) and Destination (j) regions of regional path $p=\{124\}$ also as a function of the traffic conditions in regions 2 and 3 . The average trip lengths $\bar{L}_{r p}$ are listed in meters. 
free-flow conditions, the virtual trips suffer some detours. To go from region 1 to 4 , the virtual trips switch to links in region 3 as their travel times are shorter than those in region 2. These virtual trips will then be associated with a different regional path, i.e. $p=\{134\}$. This decreases the significance level (i.e. $K_{p}$ ) of $p=\{124\}$, influencing the average trip lengths along the regions of this path.

In summary, in free-flow conditions, the detour ratios of the time-dependent virtual trips due to changes in the traffic conditions, do not influence $K_{p}$ and the average trip lengths. However, these detours make a clear difference once the regions become more congested. We also observe that both the average trip length and $K_{p}$ of an internal path are less sensitive to changes in the traffic conditions than those of a regional path. We show in the next sections, that this is well captured by the traffic-dependent framework we defined in Sect. 2.2.

\subsection{Calibration of $N_{\text {od }}$ and $S$}

In this section, we investigate how our methodology performs to estimate traffic-dependent trip lengths on a static scenario, i.e. without considering the application of a traffic model to simulate congestion. We consider the trip lengths for the regional path $p=\{124\}$ and for 300 sets of possible mean speeds $\left(v_{r}^{*}, \forall r=1, \ldots, 4\right)$ in the four regions of the L6 network (Fig. 5 (a)). Each sampled set represents different observed traffic dynamics in the regions. The sampled points vary between the free-flow speed of each region $\left(v_{r}^{f f}\right)$ and a minimum speed of $2 \mathrm{~m} / \mathrm{s}$. For this, we also consider a different number of od pairs $N_{o d}$ for the virtual set of trips and a different number of points $S$ for discretizing the speed-MFDs to construct the multi-dimensional numerical grid.

The average trip lengths strongly depend on the number of trips, $N_{o d}$, considered to construct the virtual set of trips (Batista et al., 2019). One possibility would be to consider the full set of possible combinations of od pairs in regions 1 and 4. This yields a set of $N_{o d}=5658$ virtual trips that need to be calculated for each point of the discretized fourdimensional space of the L6 network. This is still attainable for such a small network partitioned into a low number of regions (i.e. four). However, it is unfeasible from a pragmatic perspective for larger city networks partitioned into a larger number of regions. Instead, we consider a subset of $N_{\text {od }}$ pairs. This value needs to be properly set (Batista et al., 2019, in press.). We use four $N_{o d}$ values: 100, 200, 300 and 400.

We also need to discretize the speed-MFDs into $S$ points for constructing the multi-dimensional grid. As discussed in the previous section, the average trip lengths are more sensitive to the regional traffic dynamics in the congested rather than in the free-flow regime. The speed-MFDs are then discretized into a different number of points in the freeflow and congested branches. We discretize the free-flow branch of the speed-MFDs (Fig. 5 (b)) into two intervals of length $\Delta h=\frac{v_{r}^{f f}-v_{r}^{\text {crit. }}}{2}$. The congested branch is discretized with $S-1$ points, leading to $S$ intervals of length $\Delta h=\frac{v_{r}^{\text {crit. }}}{S}$. We set three values of $S$, ranging from 3 to 5 .

There are a total of 12 possible combinations of $N_{\text {od }}$ and $S$ values previously set. The goal is to investigate how our methodology performs on the estimation of the trip lengths for the regional path $p=\{124\}$ for each combination of $N_{o d}$ and $S$ values. For this, we follow the procedure depicted in Fig. 6. The only difference is that we sample 300 trials of possible observed mean speeds $\left(v_{r}^{*}, \forall r=1, \ldots, 4\right)$, within the range between $2 \mathrm{~m} / \mathrm{s}$ and the free-flow speed of each of the four regions, instead of performing an MFD simulation. This permits to mimic of different traffic dynamics in the regions. The subset of od pairs is fixed for the same value of $N_{o d}$ and this experiment. This means that we only sample once the subset of $N_{\text {od }}$ od pairs. That way, we can keep the consistency between the different 300 trials of observed mean speeds. For the benchmark approach, we determine the trip lengths $\left(L_{r p}\right)$ by directly calculating the time-dependent virtual trips for each trial of observed speeds $\left(v_{r}^{*}, \forall r=1, \ldots, 4\right)$. We then apply the developed methodology to estimate the distributions of travel distances. We determine the time-dependent virtual trips for the fixed set of $N_{o d}$ od pairs and for each point in the multi-dimensional numerical grid; so we can construct the library of time-dependent trip lengths. Based on this, we estimate the distributions of trip lengths $\left(\hat{L}_{r p}\right)$ for the regional path $p=\{124\}$, for all 12 possible combinations of $N_{o d}$ and $S$ values. Then, we determine the relative error $\varepsilon$ between the estimated distributions of trip lengths $\left(\hat{L}_{r p}\right)$ and the ones determined for the benchmark approach $\left(L_{r p}\right)$, for each region $r$ of path $p=\{124\}$ :

$$
\varepsilon=\frac{\hat{L}_{r p}-L_{r p}}{L_{r p}} \times 100 \%
$$



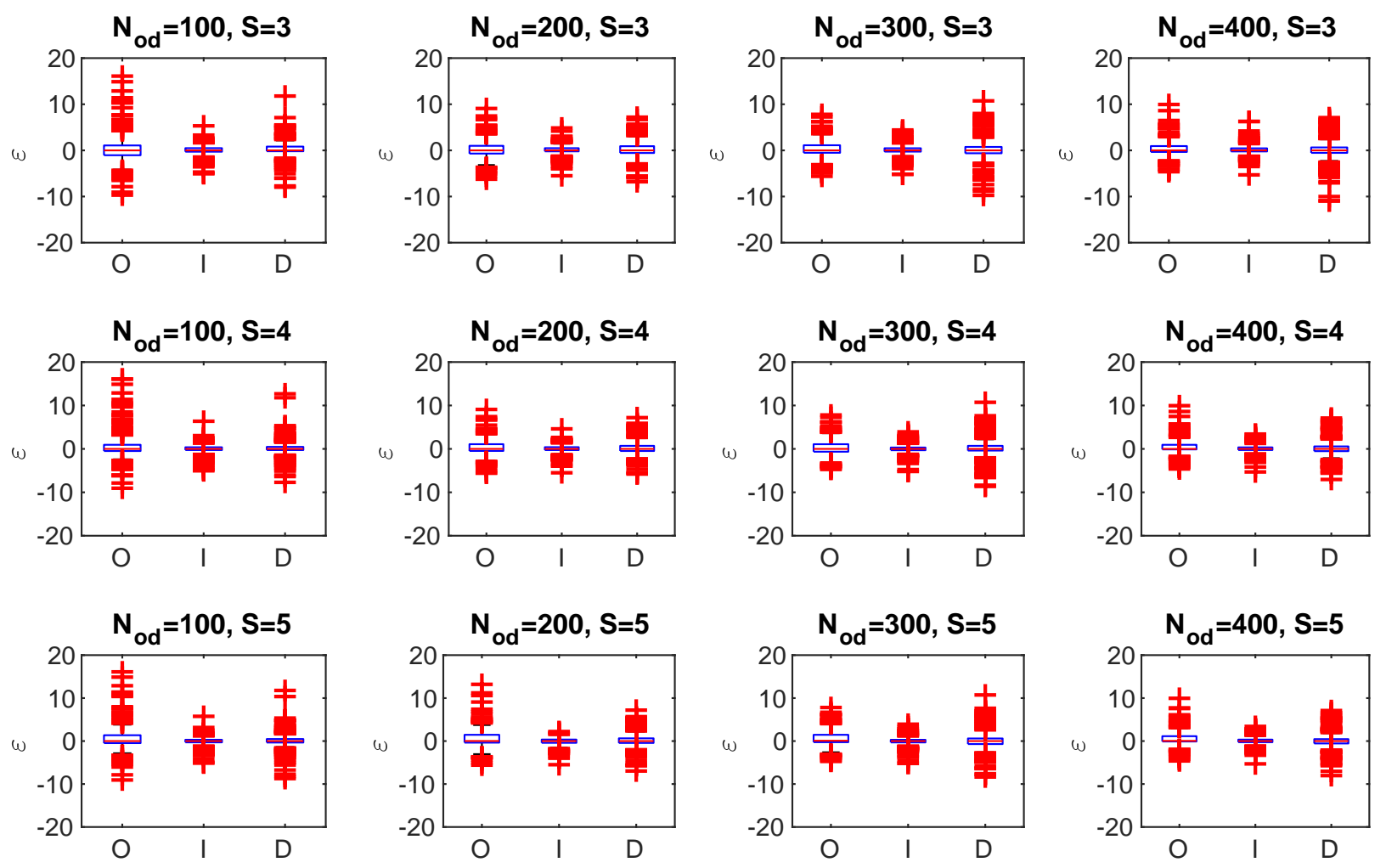

Figure 8: Relative differences $\varepsilon$ on the average trip lengths for the Origin (O), Intermediate (I), and Destination (D) regions. The results are depicted for all combinations $N_{\text {od }}$ and $S$ values considered.

Ideally, $\varepsilon$ should have a low value close to $0 \%$. This means that the estimated distributions of trip lengths $\hat{L}_{r p}$ should be close to the ones determined directly through the recalculation of the time-dependent virtual trips, i.e. $\hat{L}_{r p} \approx L_{r p}$. Fig. 8 depicts the box-and-whisker diagrams of the relative differences $\varepsilon$, for all 12 combinations of $N_{o d}$ and $S$. The horizontal red lines represent the median of the $\varepsilon$ distribution, while the red points represent the outliers. From Fig. 8, one can observe that the median of the $\varepsilon$ distributions are close to $0 \%$. Moreover, the interquartilerange of these distributions is highly centered around the medians, which shows that the relative errors are relatively small for all $N_{o d}$ and $S$ values. This confirms the good performance of the developed methodological framework for estimating the distributions of trip lengths compared to the benchmark approach. However, we can observe the presence of several outliers. To better understand the source of these outliers, we analyze the relationship between the relative error $\varepsilon$ and the number of virtual trips identified to estimate the trip length distribution, and then $\hat{L}_{r p}$. Recall that our methodology consists of identifying the $R$ closest points of the multi-dimensional numerical grid in the vicinity of the observed mean speeds $v_{r}^{*}, \forall r=1, \ldots, 4$. We then filter all the $\mu$ common virtual trips of all of these sets. We estimate the trip lengths $\hat{l}_{r p}$ for all of the identified common trips, and update the trip length distribution $\hat{L}_{r p}=\left\{\hat{l}_{r p}\right\}, \forall r \in p \wedge \forall p \in \Omega^{O D}$ that has length $\mu$. Fig. 9 depicts the relation between the relative error $\varepsilon$ and the length of the estimated trip length distribution $\mu$, for the Origin, Intermediate, and Destination regions of path $p=\{124\}$. These results include all the estimated trip lengths for all 12 combinations of $N_{o d}$ (i.e. $\left.N_{o d}=100,200,300,400\right)$ and $S$ (i.e. $S=3,4,5$ ) values. One can observe that dispersion of the relative error $\varepsilon$ reduces as the number of common trips $\mu$ increases. Low values of common virtual trips $\mu$ might not be statistically significant, leading to possible large relative errors $\varepsilon$. This explains the outliers of the box-and-whisker diagrams shown in Fig. 8.

Overall, the previous analysis guides on how to properly select the $N_{o d}$ and $S$ values. The results depicted in Fig. 8 show that the developed methodology performs well when estimating the time-dependent distributions of travel 

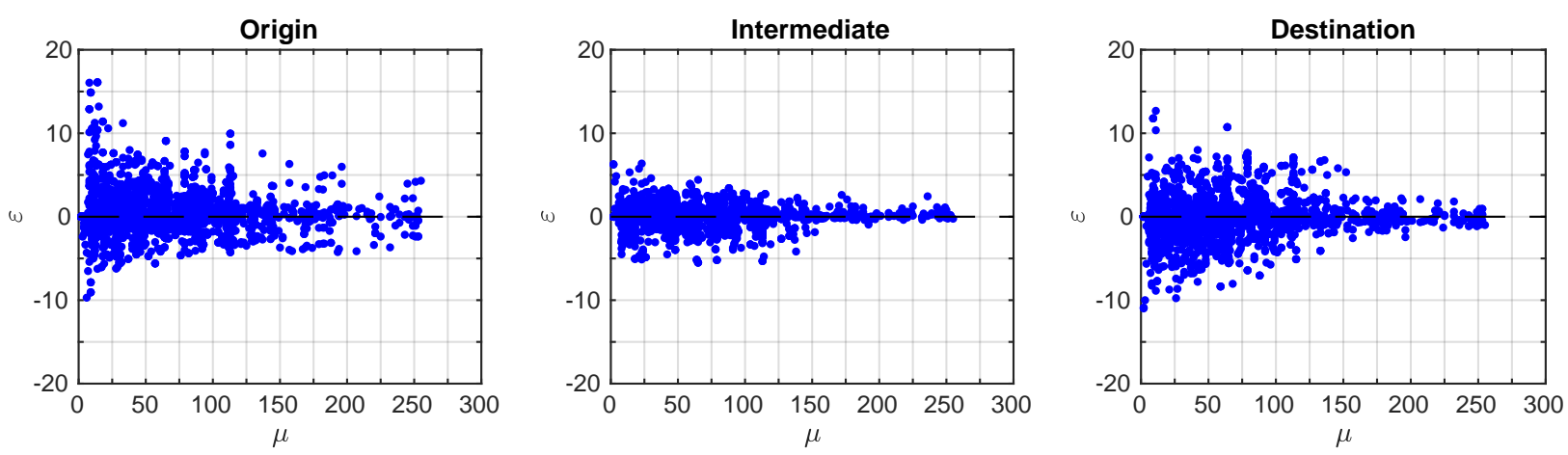

Figure 9: Relative error $\varepsilon$ as a function of the number of common trips $\mu$, for all 12 combinations of $N_{o d}$ and $S$. The results are depicted for the Origin, Intermediate and Destination regions of path $p=\{124\}$. The horizontal black dashed line indicates a reference point at $\varepsilon=0 \%$.

distances even for low $N_{o d}=100$ and $S=3$ values. However, this performance depends on the total number $\mu$ of common trips as shown in Fig. 9. By setting a small value of $N_{o d}$, t can happen that one cannot identify a statistically significant number of common virtual trips $\mu$. By increasing $N_{o d}$, one might avoid the previously mentioned limitation. However, this increases the computational cost required to build the uniformly distributed multi-dimensional grid as one also increases the total number of shortest-trips in time to compute for each point of the grid.

We also analyze the computational time required to estimate the distributions of trip lengths using the developed methodological framework, and by the benchmark approach which requires the direct calculation of the trafficdependent shortest-trips in time. Fig. 10 depicts the box-and-whisker diagrams of the computational times taken by developed methodology and the benchmark approach. As one can observe, our methodology requires a much lower computational time. The median computational time required by the developed methodology was 0.093 [s], compared to 0.48 [s] for the benchmark approach. Therefore, the developed methodological framework to determine the trafficdependent trip lengths not only provides accurate estimations but also requires a much lower computational time than if one has to recalculate the shortest virtual trips in time.

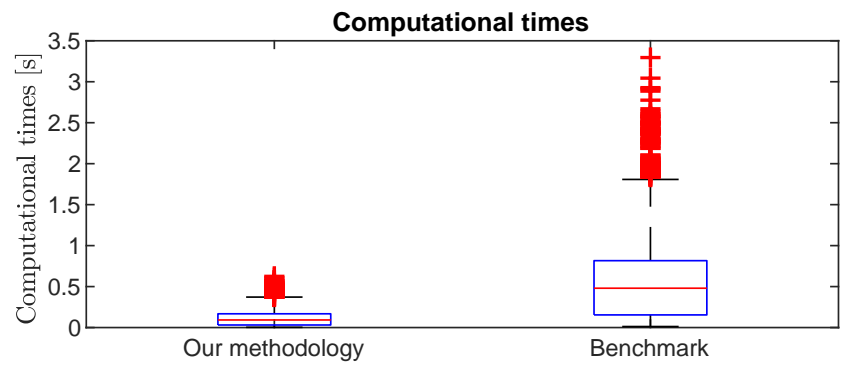

Figure 10: Evolution of the IQR values as a function of $S$, for the Origin, Intermediate and Destination regions of path $p=\{124\}$. The results are depicted for $N_{o d}=100,200,300,400$.

In this section, we also investigate the validity of the assumption made in Sect. 2.2. We assumed that the topology of the $L_{r p}$ function (defined in Eq. 4) can be approximated by a hyper-plane in the vicinity of $v^{*}$ in the R-dimensional space. We focus on the coefficient of determination calculated for fitting the $L_{r p}$ function, and then predict $\hat{l}_{r p}$. A coefficient of determination approximately equal to 1 , shows the existence of the multi-linear relationship between the prediction $\hat{l}_{r p}$ and the predictor variables $v_{r}^{*}$. Fig. 11 depicts the histograms of the coefficient of determination, for the Origin, Intermediate and Destination regions of path $p=\{124\}$. The results include all the estimations of the trip lengths $\hat{l}_{r p}$, for all 12 combinations of $N_{o d}$ (i.e. $N_{o d}=100,200,300,400$ ) and $S$ (i.e. $S=3,4,5$ ) values. As one can observe in Fig. 11, the coefficient of determination is 1.0 for almost all the estimations, therefore validating our initial assumption. 

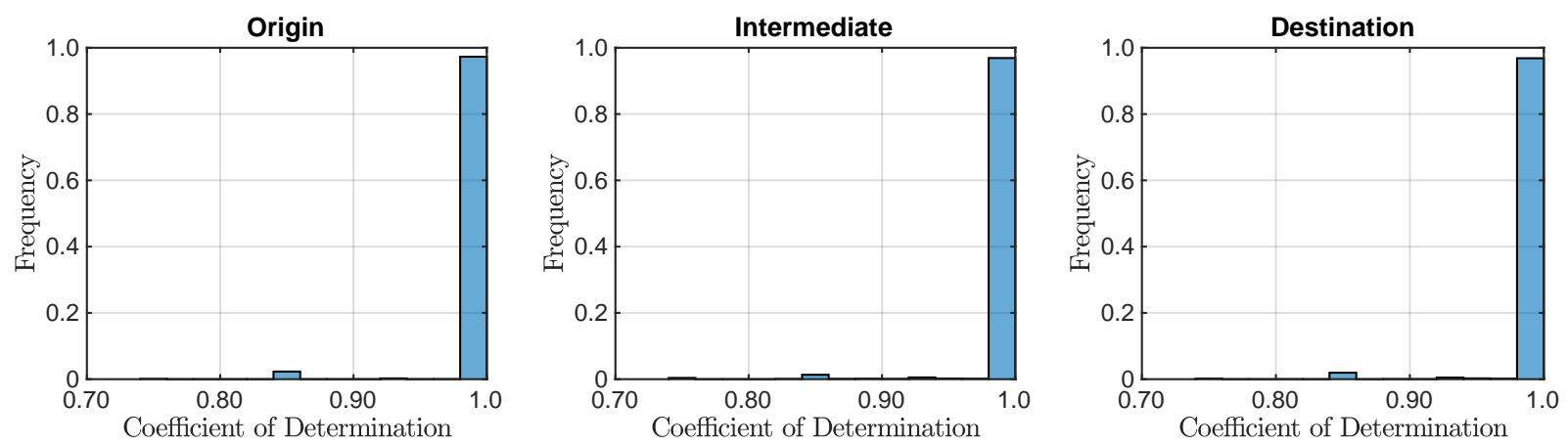

Figure 11: Histograms of the coefficient of determination, for the Origin, Intermediate and Destination regions of path $p=\{124\}$. The bin-width is 0.02

\subsection{Validation of the methodology to estimate traffic-dependent trip lengths on the L6 network}

In this section, we investigate how the methodology for estimating the time-dependent trip lengths performs in an MFD simulation environment, on the L6 network. This permits validating the developed methodology to estimate the traffic-dependent distributions of trip lengths against the benchmark approach discussed in Fig. 6. Moreover, based on the discussion of the previous section, we set $N_{o d}=200$ and $S=5$.

Fig. 12 and Fig. 13 depict the evolution of the traffic states, i.e. mean speeds $\bar{v}_{r}, \forall r=1, \ldots, 4$, for both freeflowing and congested scenarios. The results are shown for the DUE (in Fig. 12) and SUE (in Fig. 13) conditions of the network. We model the traffic states for three different cases to determine the trip lengths. In the first one, we determine the trip lengths based on the shortest-trips in terms of distance, i.e. static trip lengths. The results are represented by the blue curve in Fig. 12 and by the red curve in Fig. 13, for the DUE and SUE conditions, respectively. In the second case, we estimate the trip lengths based on the developed methodology for each sub-interval $\delta t$ in the outer-loop of the R-DTA, see Fig. 2. We refer to this case as the estimated one. The results are represented by the green and gold color curves in Fig. 12 and Fig. 13, for the DUE and SUE respectively. In the third case, we update the time-dependent trip lengths in the outer loop based on the R-DTA based on the calculation of the virtual trip set, given the observed traffic conditions, i.e. $v_{r}^{*}, \forall r=1, \ldots, 4$, from the previous period $\delta t$. Recall that this represents the benchmark scenario, which is represented by the black dashed curves in Fig. 12 and Fig. 13. This third case is set up as the reference, but the computational costs are large, and it is not reasonable to apply it in practice. However, we consider it here just for validation purposes. For both the DUE and SUE conditions, we fix the choice set, and path flows for the benchmark approach, based on the results from the estimated case.

We first analyze the traffic states in the regions predicted for the static trip lengths. For the free-flow scenario, and under DUE conditions, all drivers traveling on OD14 choose regional path $p=\{124\}$. The only two exceptions occur at $t=2200$ and $t=2600$ seconds, where drivers equally choose paths $p=\{124\}$ and $p=\{134\}$. One can observe that the mean speed $\bar{v}_{1}$ decreases between $\sim 2000$ and 4000 seconds. This is due to an increase in the demand traveling on OD14 during this period. After this period, the speed $\bar{v}_{1}$ slightly increases to $\sim 4.5 \mathrm{~m} / \mathrm{s}$. Between 4000 and 6000 seconds, there is a demand peak on OD23. The demand of this OD is assigned interchangeable in the proportions of $55 \%$ and $45 \%$ to the regional paths $p=\{23\}$ and $p=\{213\}$, between consecutive assignment periods. This leads to small increases and decreases of the mean speeds in region 1 that are offset between consecutive assignment periods depending on which path is assigned the larger fraction of the demand. These fluctuations of the mean speed are also observed in regions 2 and 3, between 2000 and 3000 seconds. They are also offset. For example, at $t=2200$ seconds, drivers also choose to travel on path $p=\{134\}$. This leads to an increase of $\bar{v}_{2}$, and a decrease of $\bar{v}_{3}$ at this time instant. After $\sim 3500$ seconds, we observe a decrease of $\bar{v}_{2}$, and $\bar{v}_{3}$. This is thanks to the demand increase traveling on OD23. In region 4, between $\sim 2000$ and 3500 seconds, the demand peak of drivers traveling on OD14 starts to arrive at their destination, leading to a reduction of $\bar{v}_{4}$. In the case of the SUE, $\sim 65 \%$ of the OD14 demand is assigned to path $p=\{124\}$, while the remaining is assigned to $p=\{134\}$. In the case of the OD23 demand, $\sim 55 \%$ is assigned to $p=\{23\}$. The remaining vehicles are assigned to $p=\{213\}$. The path flow distributions remain approximately constant over the whole simulation period, leading to the disappearance of the fluctuations present on $\bar{v}_{1}, \bar{v}_{2}$ and $\bar{v}_{3}$ in the DUE conditions. In contrast, in the congested scenario, the fluctuations increase for the DUE conditions in 
(a) Region 1: free-flow

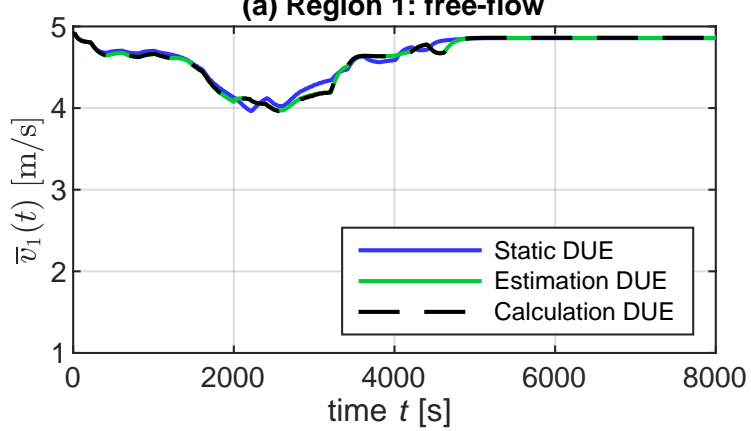

(c) Region 3: free-flow

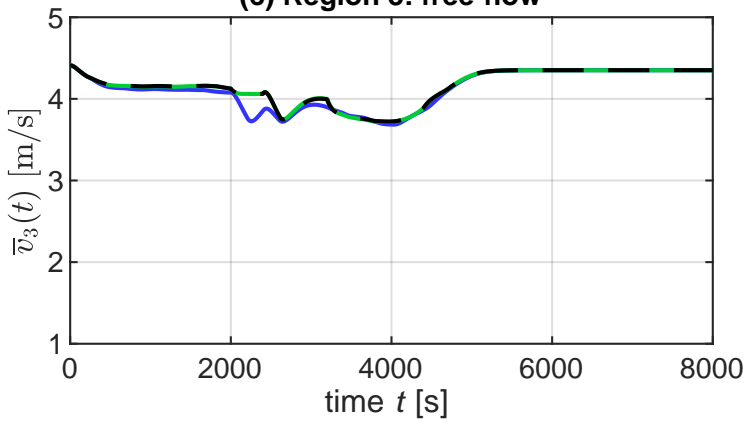

(e) Region 1: congested

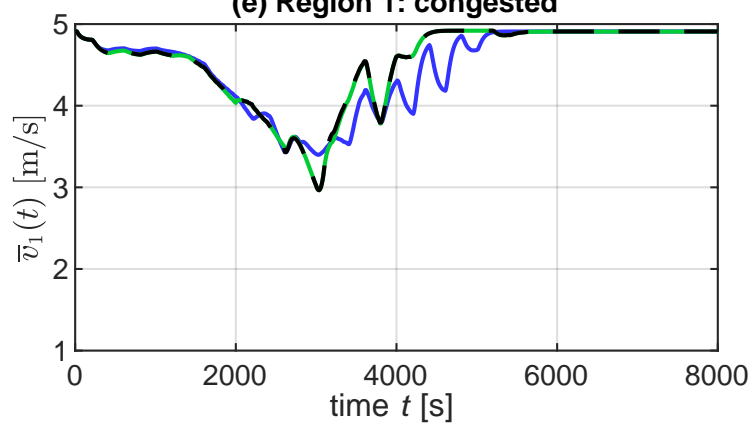

(g) Region 3: congested

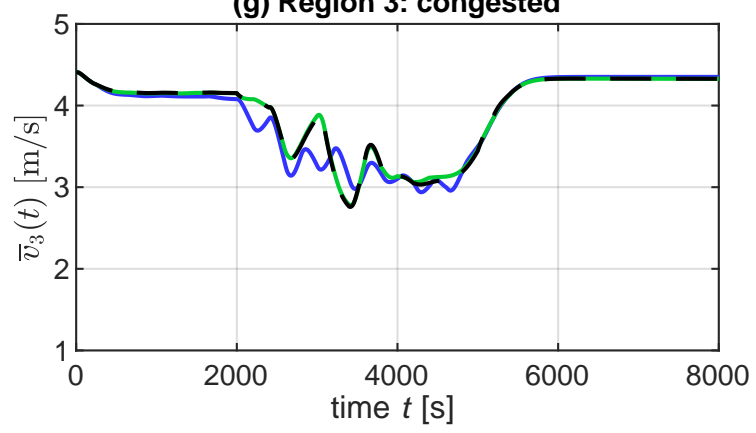

(b) Region 2: free-flow

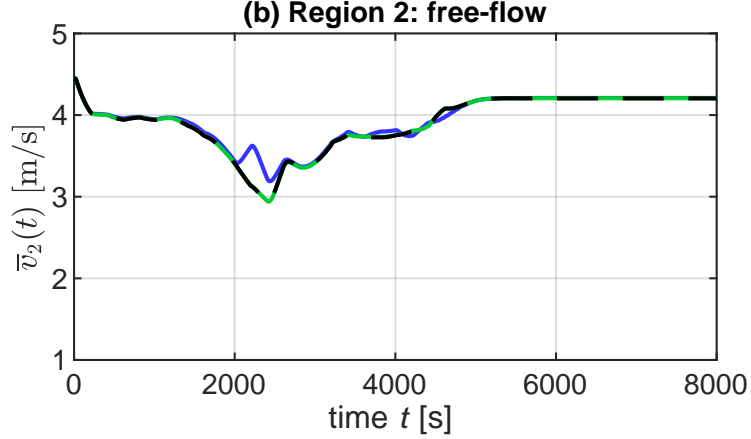

(d) Region 4: free-flow

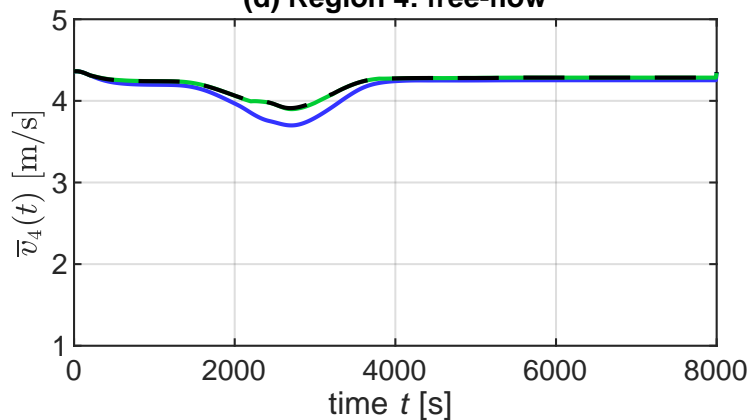

(f) Region 2: congested

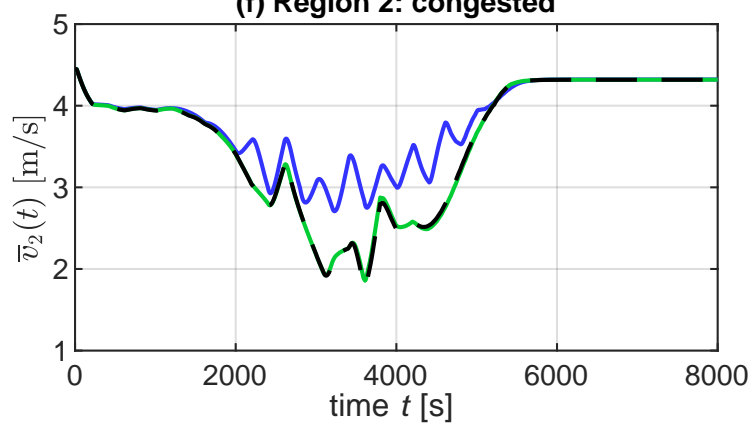

(h) Region 4: congested

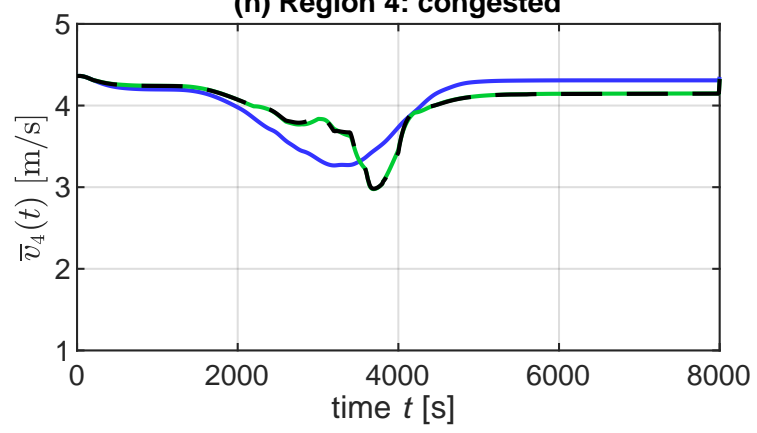

Figure 12: Evolution of the regional mean speeds $\bar{v}_{r}, \forall r=1, \ldots, 4$ in the regions of the L6 network for the free-flow (a-d) and congested (e-h) scenarios. The results are depicted for the static (blue and gold curves), estimated (green and red curves), and calculated (black dashed curves) trip lengths, and for the DUE. 
(a) Region 1: free-flow

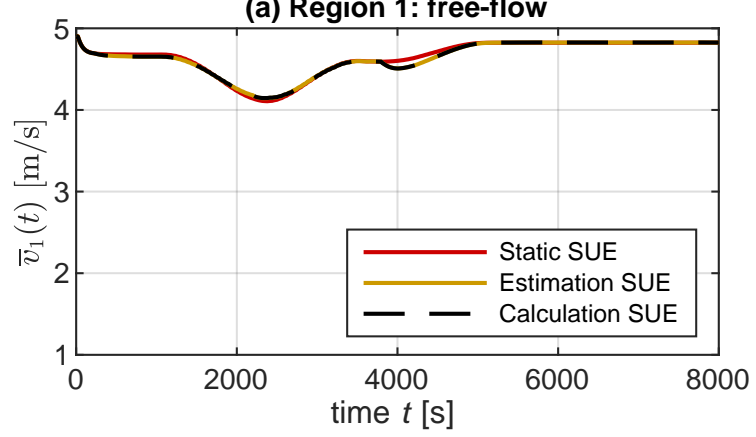

(c) Region 3: free-flow

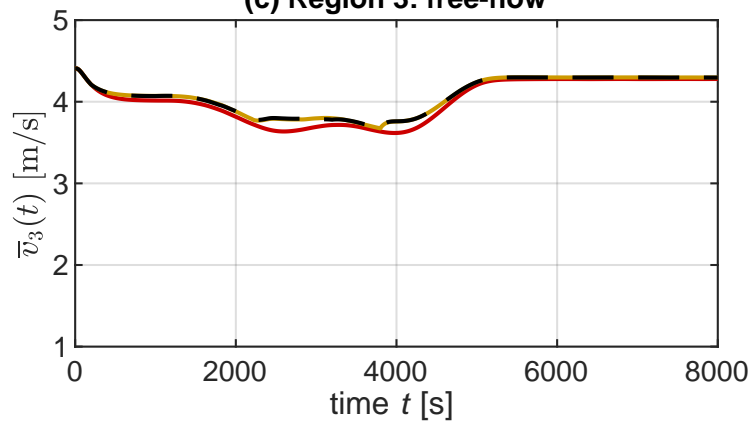

(e) Region 1: congested

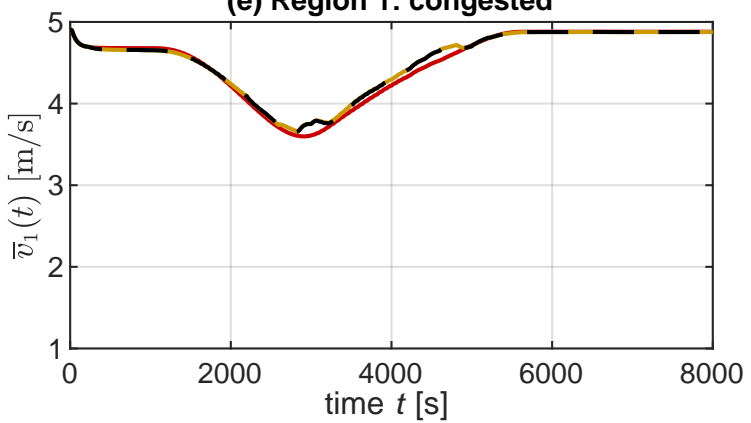

(g) Region 3: congested

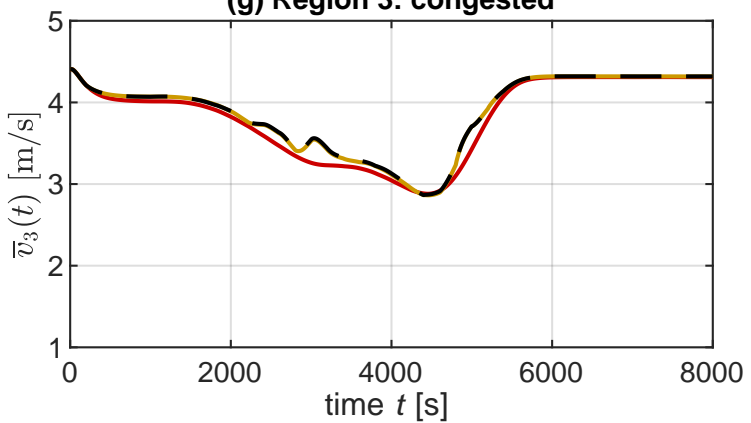

(b) Region 2: free-flow

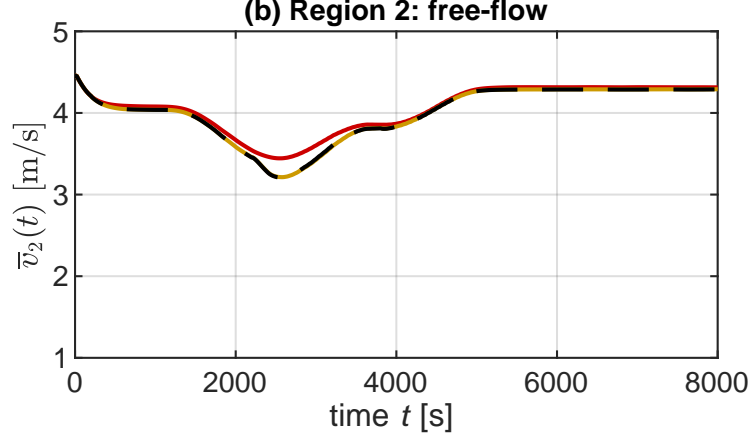

(d) Region 4: free-flow

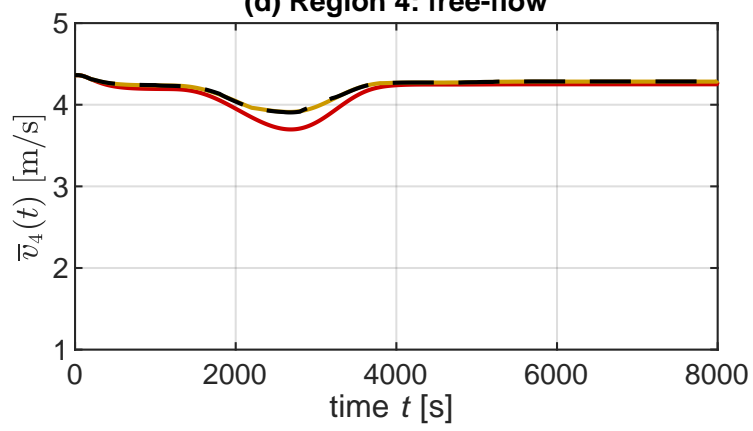

(f) Region 2: congested

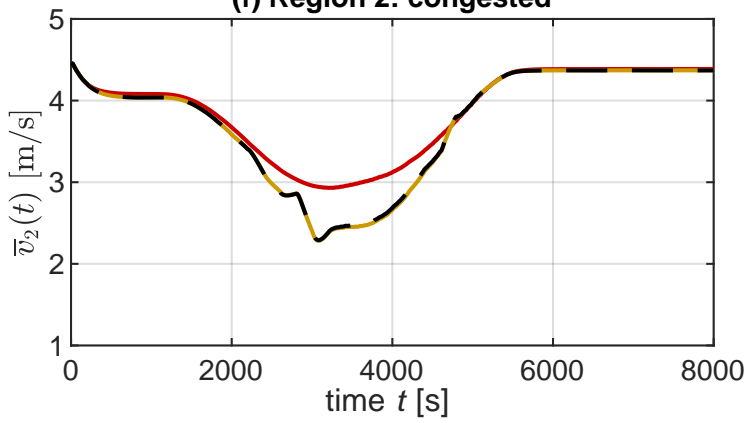

(h) Region 4: congested

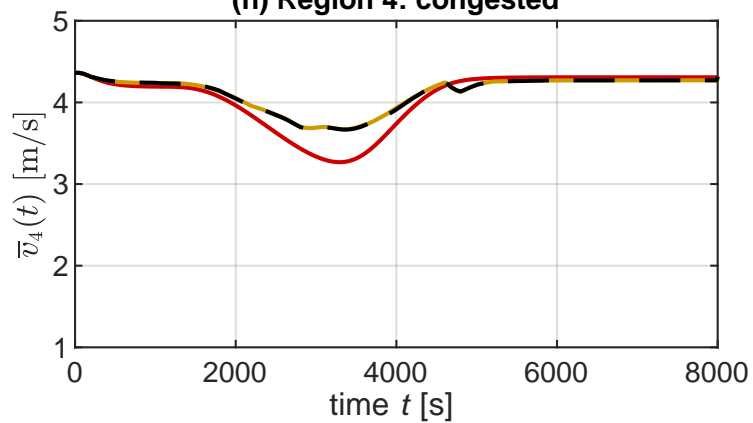

Figure 13: Same as in Fig. 12, but for the SUE. 
regions 1, 2, and 3. Between $~ 2000$ and 4000 seconds, the demand of OD14 is assigned interchangeably in the proportions of $95 \%$ and $5 \%$ to the paths $p\{124\}$ and $p=\{134\}$. This explains the lagged increase and decrease of the mean speed in regions 2 and 3. The fluctuations observed in region 1, are explained by the different distances traveled by drivers on both paths and in region 1 . The average trip length for $p\{124\}$ in region 1 is 280 meters, while for $p\{134\}$ in region 1 is 342 meters. Drivers traveling on path $p\{134\}$ need more time to complete their trips in region 1 , than the ones traveling on path $p\{124\}$. Then, when regional path $p\{134\}$ is chosen, the accumulation in region 1 increases for a longer period, leading to a larger decrease of the mean speed $\bar{v}_{1}$. Also, in the congested scenario, for the static trip lengths and under SUE conditions, there are fours paths chosen by drivers: $p\{124\}, p\{134\}, p\{23\}$ and $p\{213\}$. Similarly to the static SUE case, in the free-flow scenario, the path flows remain approximately constant over the whole simulation period, and the fluctuations vanish.

The results depicted in Fig. 12 and Fig. 13 also highlight the importance of updating the trip lengths according to the changes in the traffic conditions over time. This is also in line with the discussion by Yildirimoglu and Geroliminis (2014). We observe that in free-flow scenarios, the differences between the predicted traffic conditions for the static and the time-dependent trip lengths are not significant. However, these differences become evident when the congestion level in the regions increases.

We now analyze how the estimation methodology performs in an MFD-simulation environment. For this, we compare the evolution of the traffic states between the estimated distributions of trip lengths and the benchmark approach. We observe in Fig. 12 and Fig. 13 that the predicted traffic states for the estimated trip lengths (green and gold color curves) match the evolution trend of the recalculated trip lengths (black dashed curves). This is true for both free-flow and congested scenarios and when the network is under DUE and SUE conditions.

We also determine the relative error $\varepsilon$ for the Origin, Intermediate, and Destination regions of all chosen paths, using Eq. 11. In the case of the trip length distributions, we calculate the relative differences $(\Phi)$ between the estimated trip length sample $\left(\hat{l}_{r p}\right)$ and the one determined directly by the recalculation of the shortest virtual trip in terms of time $\left(l_{r p}\right)$ :

$$
\Phi=\frac{\hat{l}_{r p}-l_{r p}}{l_{r p}} \times 100 \%
$$

Fig. 14 depicts the box-and-whisker diagrams of the relative differences $\varepsilon$ and $\Phi$, for the Origin (O), Intermediate (I) and Destination (D) regions of all used paths during the simulation period. The results are shown for the free-flow and congested scenarios and when the network is under DUE and SUE conditions. The horizontal red lines represent the median of the distributions; they are all close to $0 \%$. One can also observe that the distributions are narrow around $0 \%$, highlighting the good performance of the estimation methodology for the trip lengths. The estimation methodology performs worse for estimating the trip lengths for the Destination regions, as highlighted by the presence of some outliers. Nevertheless, we reinforce the fact that the distributions of the relative errors for the Destination regions are narrow around $0 \%$. The standard deviations range between $1 \%$ to $2 \%$ for the Origin and Intermediate regions and between $4 \%$ to $8 \%$ for the Destination regions.

\section{Validation of the methodology to estimate traffic-dependent trip lengths on medium-sized city network}

In the same spirit as the previous section, we do similar testing of the proposed methodology for estimating the trip lengths in an MFD simulation environment, but for a medium-sized network and more complex demand scenarios. The medium-sized network is depicted in Fig. 15 (a), and includes the $3^{\text {rd }}$ and $6^{\text {th }}$ districts of Lyon and the city of Villeurbanne (France). We refer to this network as L63V. It contains 3127 nodes and 3363 links. It is partitioned into seven regions, for which we fitted the speed-MFDs depicted in Fig. $15(\mathrm{~b})$. The free-flow speeds are $v_{r}^{f f}=$ $\{5.2,6.5,5.8,5.5,5.4,7.0,6.0\}, \forall r=1, \ldots, 7$, while the critical speeds are $v_{r}^{c r i t .}=\{2.6,3.3,2.9,2.8,2.7,3.5,3.0\}, \forall r=$ $1, \ldots, 7$. Again, in this section, the speeds are expressed in $\mathrm{m} / \mathrm{s}$. The calibration of the speed-MFDs and partitioning of the city network follow Batista and Leclercq (2020). Fig. 15 (c) depicts the demand scenarios. We consider a total of 6 OD pairs: $1-2 ; 2-5 ; 4-4 ; 4-7 ; 5-1$; and $6-4$. We have a total of 73 regional paths. For calculating the library of trip lengths used for the online estimation, we have calibrated $N_{o d}$ and $S$ as discussed in the previous sections. We then set $N_{o d}=200$ and $S=7$. 

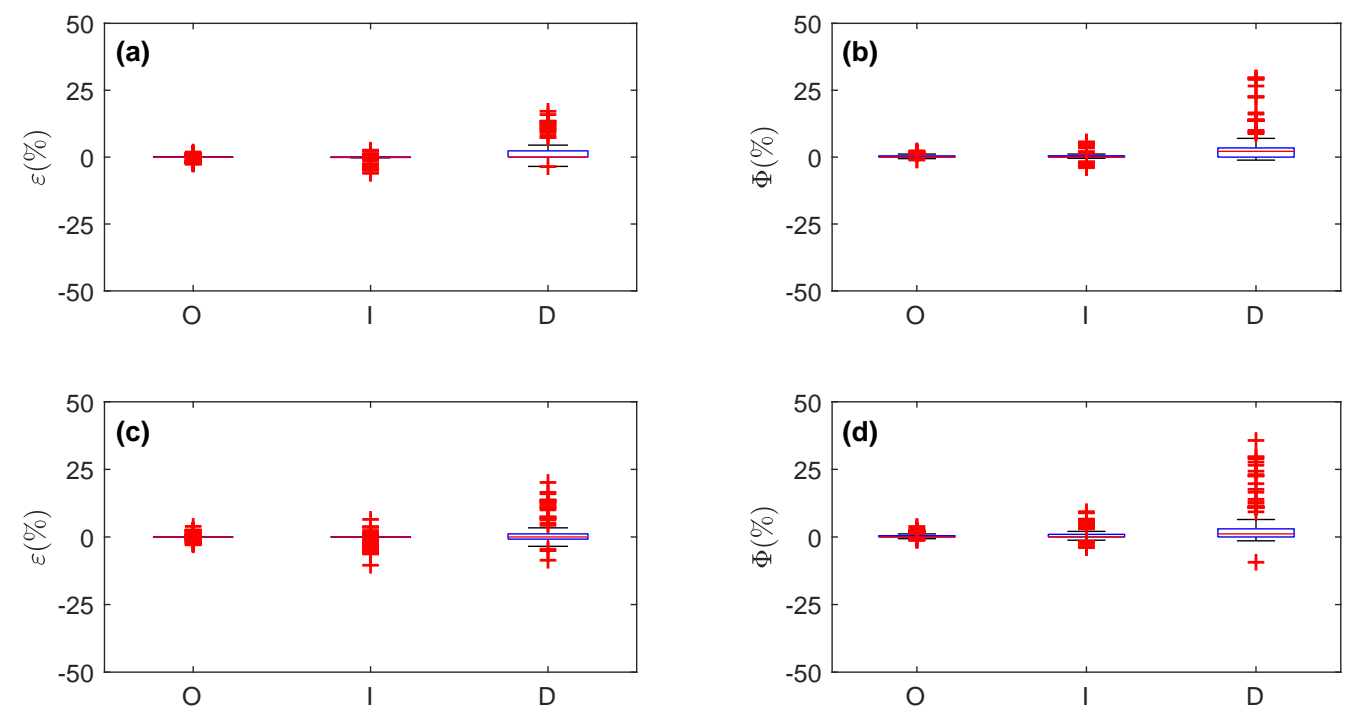

Figure 14: Relative differences $\varepsilon$ for the DUE and the (a) free-flow and (c) congested scenarios. Relative differences $\Phi$ for the SUE and the (b) free-flow and (d) congested scenarios.
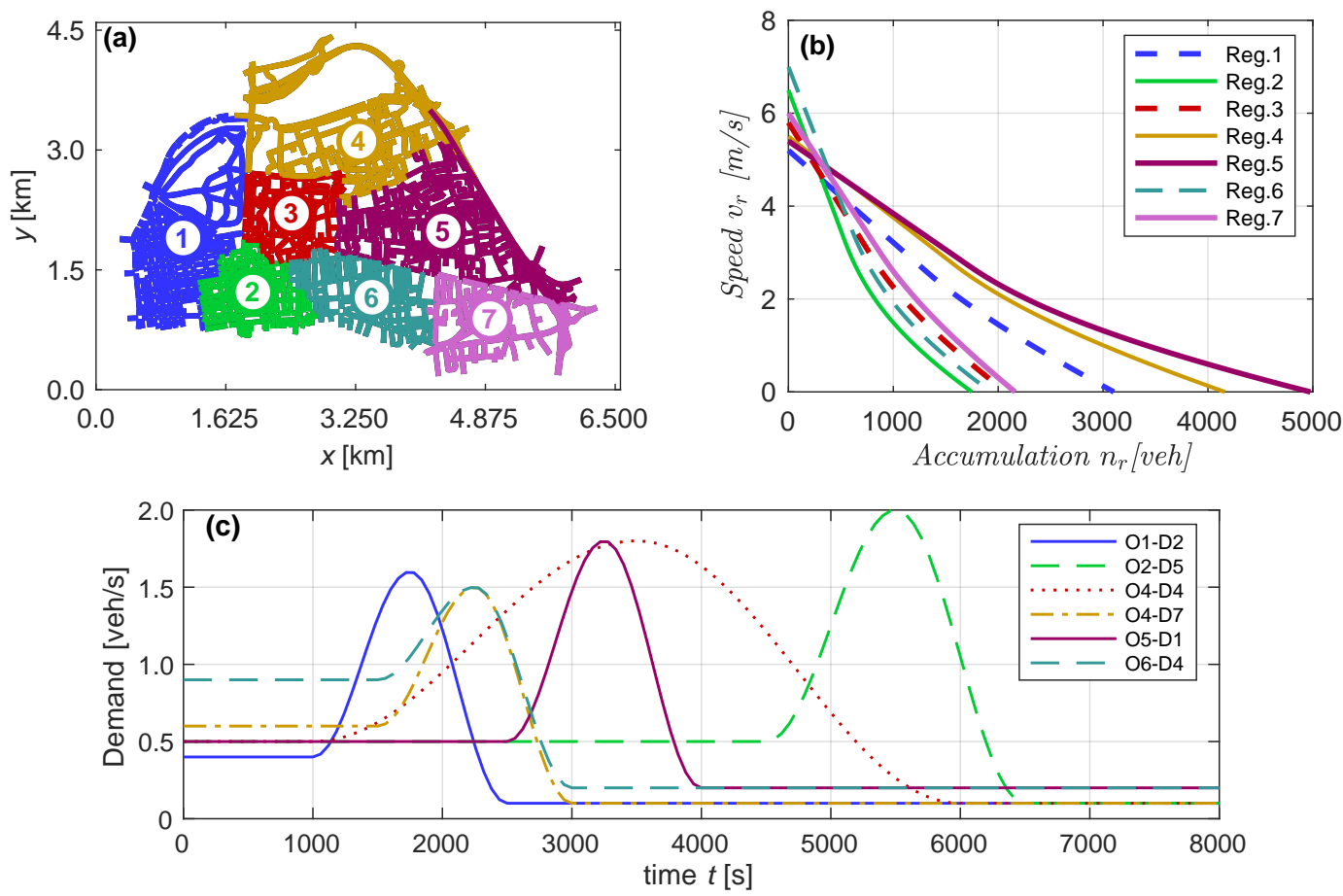

Figure 15: (a) $3^{\text {rd }}$ and $6^{\text {th }}$ district Lyon network and the city of Villeurbarnne (L63V) divided into seven regions. (b) Speed-MFDs of the L63V network. (c) Demand scenarios.

Fig. 16 and Fig. 17 depict the evolution of the traffic dynamics in the regions of the L63V network, for the DUE

and SUE conditions, respectively. The blue and red curves represent the results predicted for the static trip lengths and the network under DUE and SUE conditions, respectively. The green and gold color curves represent the results for the estimated trip lengths when the network is under DUE and SUE conditions, respectively. The black dashed lines represent the traffic states predicted with the benchmark approach, where we recalculate the shortest-trips in time 
(a) Region 1: free-flow

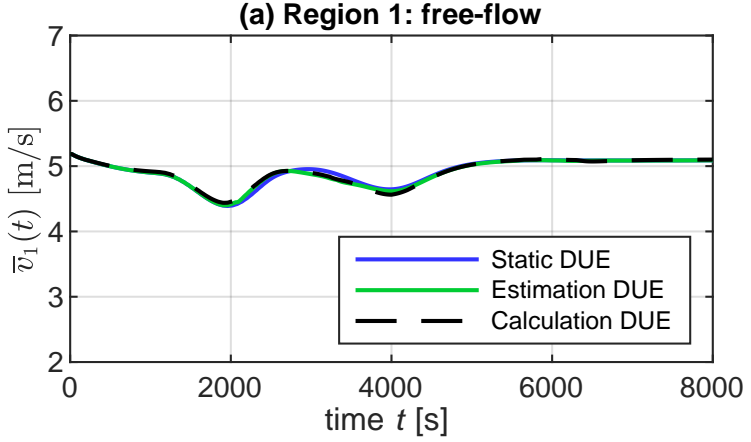

(c) Region 3: free-flow

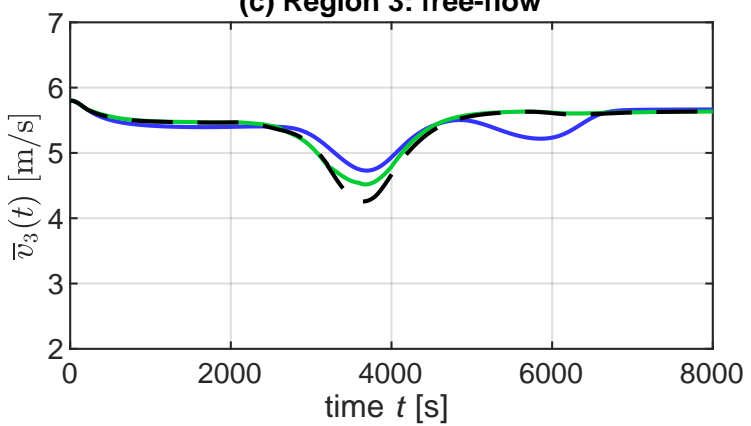

(a) Region 1: free-flow

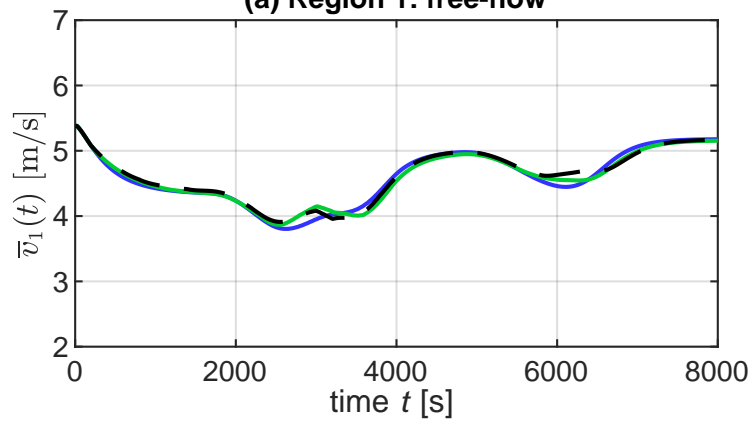

(c) Region 3: free-flow

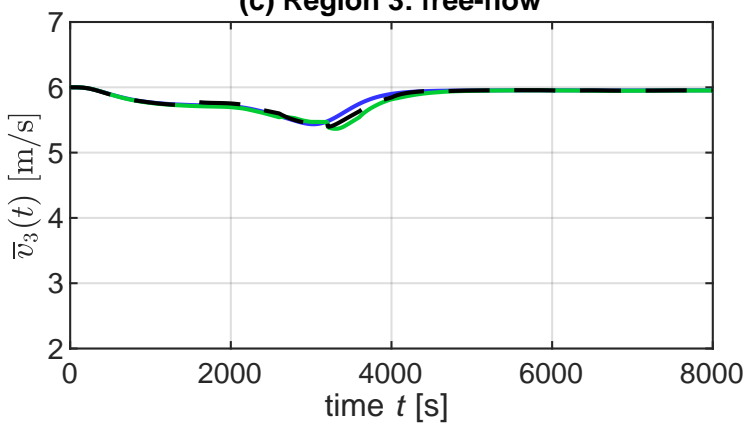

(b) Region 2: free-flow

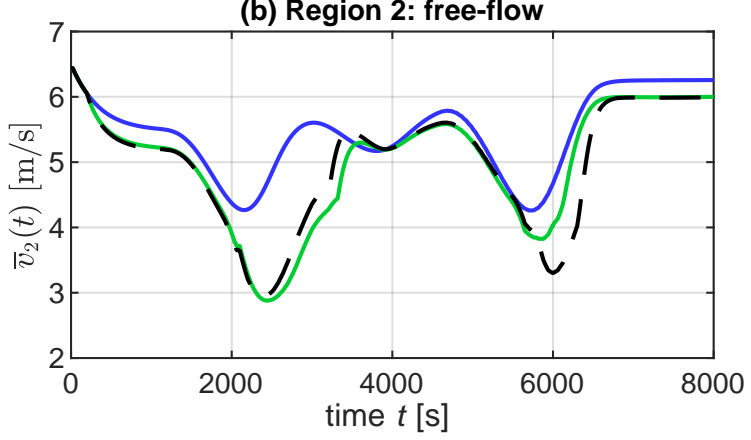

(d) Region 4: free-flow

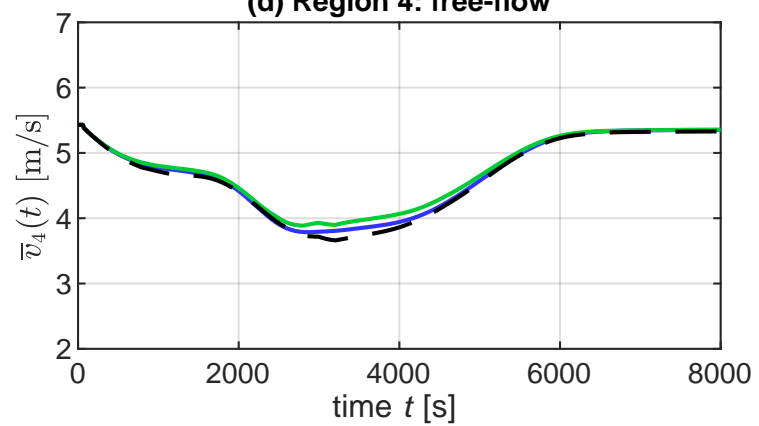

(b) Region 2: free-flow

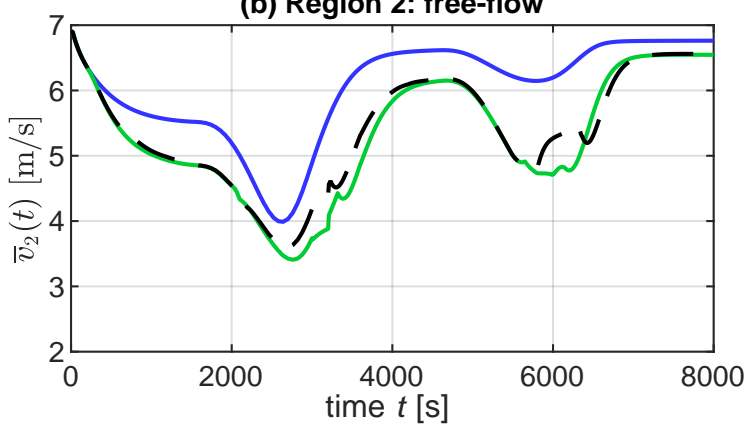

Figure 16: Evolution of the regional mean speeds $\bar{v}_{r}, \forall r=1, \ldots, 7$ in the regions of the L63V network. The results are depicted for the static (blue curve), estimated (green curve) and calculated (red curve) trip lengths, for the DUE conditions.

given the observed traffic conditions. One can observe the close evolution of the traffic states in the regions between the case when one estimates the distributions of the trip lengths and the benchmark approach. Moreover, these results also highlight the importance of updating the trip lengths according to the traffic dynamics in the regions. 
(a) Region 1: free-flow

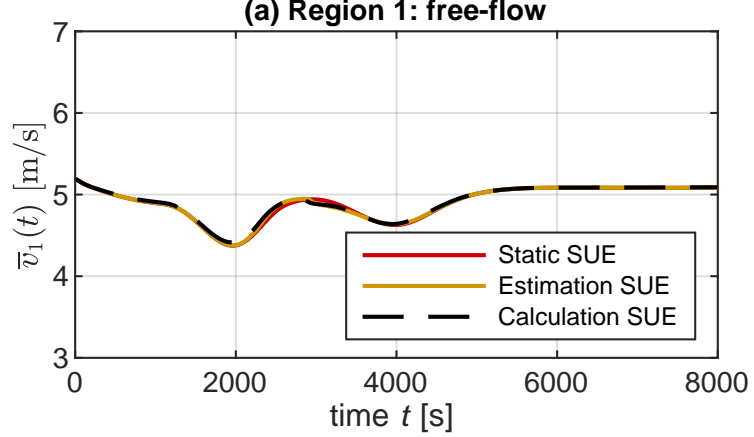

(c) Region 3: free-flow

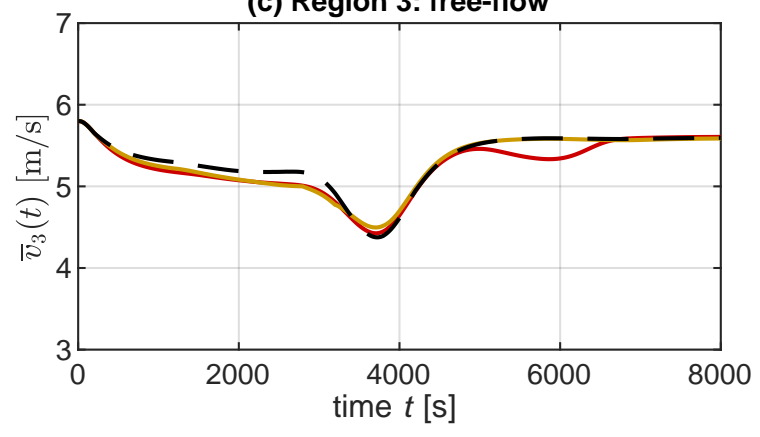

(a) Region 1: free-flow

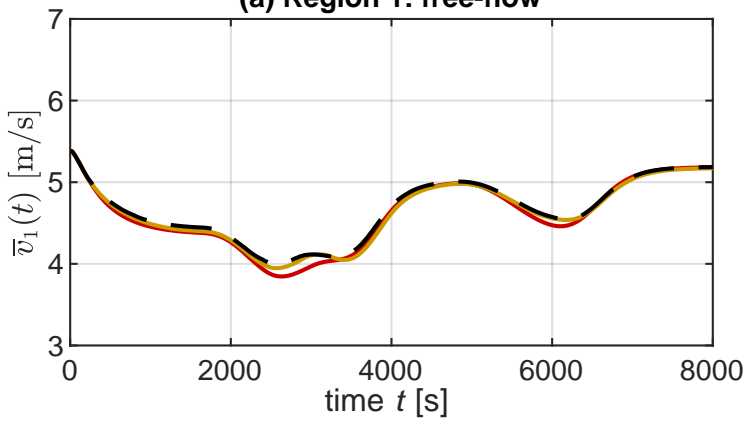

(c) Region 3: free-flow

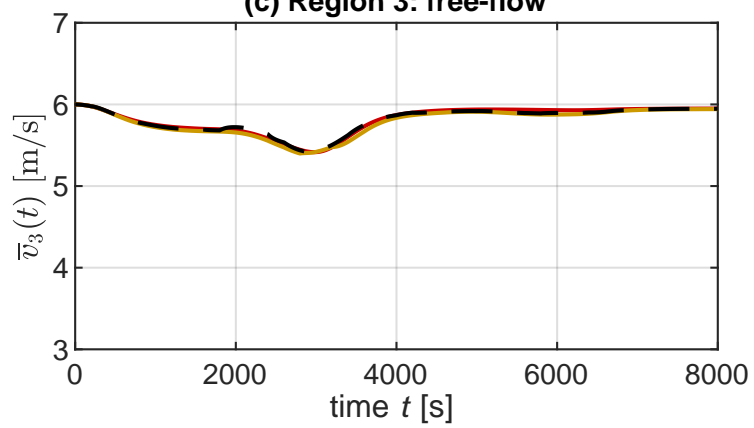

(b) Region 2: free-flow

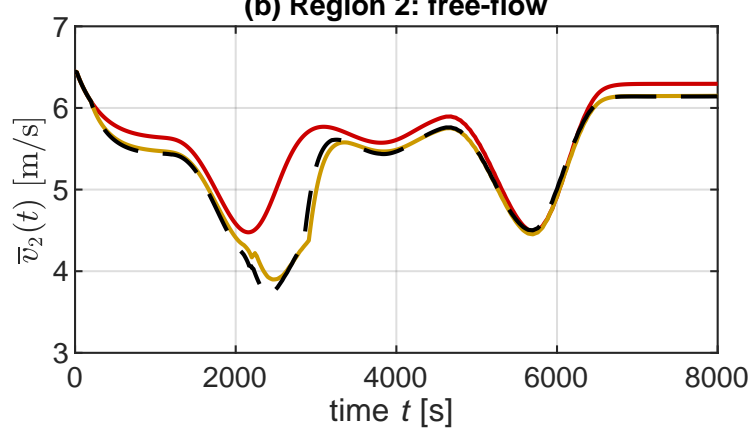

(d) Region 4: free-flow

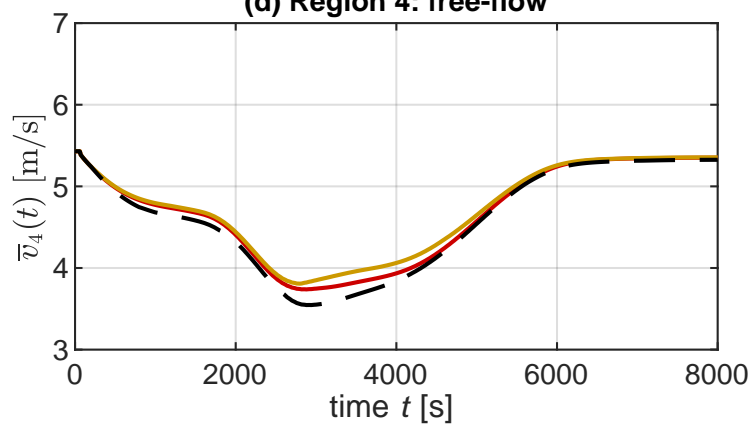

(b) Region 2: free-flow

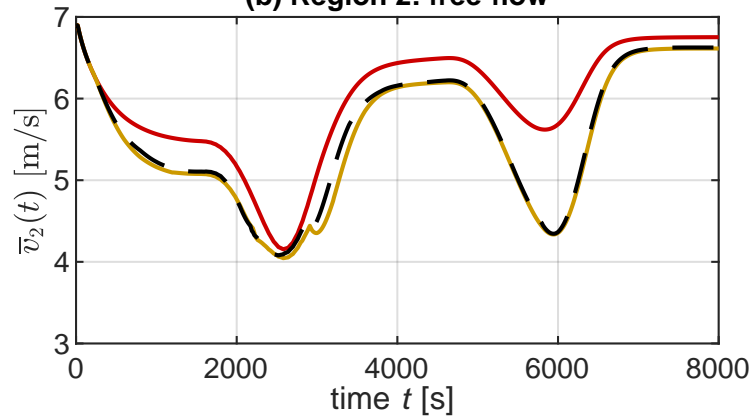

Figure 17: Same as in Fig. 16, but for the SUE conditions. 


\section{Discussion and conclusions}

In this paper, we develop a methodological framework for estimating traffic-dependent distributions of trip lengths, given the observed traffic conditions in the regions, and without the need to recalculate the set of virtual trips at each step. We discuss how this framework is incorporated in the R-DTA proposed by Batista and Leclercq (2019), to target the Deterministic and Stochastic User Equilibrium. We show that the significance level of paths and the trip lengths are influenced by the traffic dynamics in the regions. This is particularly observed for congested regions that cause detours on the shortest virtual trips in time. We validate the methodology to estimate time-dependent trip lengths on small and medium-size city networks, and in an MFD simulation environment. The estimated trip lengths allow us to predict the evolution of traffic dynamics with results similar to those from the calculation of the shortest virtual trips in terms of time. This shows the good performance of our methodological framework for estimating online traffic-dependent trip lengths for MFD-traffic models. Our simulation results also put in evidence the importance of updating the trip length distributions according to the observed traffic conditions, also following the discussion by Yildirimoglu and Geroliminis (2014). We also show that the computational time required by the developed methodological framework is much lower than the one required by a benchmark approach where one has to recalculate the set of traffic-dependent virtual trips and then the corresponding distributions of trip lengths.

The current methodology for estimating traffic-dependent trip lengths requires the construction of a multidimensional grid where for each point one has to determine a set of time-dependent set of virtual trips. This requires the proper calibration of $N_{o d}$ and $S$ points of the multi-dimensional grid, to ensure the good performance of the methodology. This permits constructing the library of distributions of trip lengths that will be used in the online estimation procedure, i.e. within an MFD-based simulation environment. Despite these calculations being done offline, they can become computationally expensive especially for large city networks, as they involve the computations of several thousands of shortest-trips in time. On the other hand, it is also important to note that the Monte Carlo procedure, used to sample the od pairs in the city network, neglects existing possible correlations between regional OD pairs. Driven by these limitations, it is desirable to develop a more robust sampling methodology of the od pairs in the city network, ensuring that: (i) the determined set is representative of the full set of possible trips connecting one OD pair; and (ii) the existing spatial correlations between regional OD pairs have been properly taken into account. This will reduce the computational costs of gathering the library of regional paths and trip lengths, allowing the further extension of our methodological framework to large city networks. Another interesting line of research is to investigate how realistic are the determined time-dependent distributions of trip lengths against real trip patterns of drivers, e.g. trip patterns from Global Positioning System trajectories. One promising idea in this direction lies in the characterization of a time-dependent trip detour ratio (Yang et al., 2018), i.e. how much the real trips of drivers deviate from the shortest-trip in distance.

\section{Acknowledgements}

The authors thank the anonymous reviewers for their critical assessment of our paper as well as for their comments and suggestions that have much improved this paper. S. F. A. Batista and M. Menéndez acknowledge support by the NYUAD Center for Interacting Urban Networks (CITIES), funded by Tamkeen under the NYUAD Research Institute Award CG001 and by the Swiss Re Institute under the Quantum Cities ${ }^{T M}$ initiative. L. Leclercq acknowledges funding by the European Research Council (ERC) under the European Unions Horizon 2020 research and innovation program (grant agreement No 646592 - MAGnUM project).

\section{Authors contributions}

S. F. A. Batista contributed to the conceptualization, methodology, validation and results analysis, and writing of the original draft of the paper. Ludovic Leclercq and Mónica Menéndez contributed to the conceptualization, methodology and review \& editing of the paper. All the authors have approved the final version of this paper submitted to publication. 


\section{References}

Aboudolas, K., Geroliminis, N., 2013. Perimeter and boundary flow control in multi-reservoir heterogeneous networks. Transportation Research Part B: Methodological 55, 265-281.

URL https : //dx.doi.org/10.1016/j.trb.2013.07.003

Ambühl, L., Loder, A., Zheng, N., Axhausen, K. W., Menendez, M., 2019. Approximative network partitioning for mfds from stationary sensor data. Transportation Research Record.

URL https : //dx.doi.org/10.1177/0361198119843264

Arnott, R., 2013. A bathtub model of downtown traffic congestion. Journal of Urban Economics 76, 110-121.

URL https ://dx.doi.org/10.1016/j.jue.2013.01.001

Batista, S., Leclercq, L., 2020. Regional dynamic traffic assignment with bounded rational drivers as a tool for assessing the emissions in large metropolitan areas. Transportation Research Interdisciplinary Perspectives 8, 100248.

URL https: //dx.doi.org/10.1016/j.trip.2020.100248

Batista, S. F. A., Cantelmo, G., Menendez, M., Antoniou, C., 2021. On the calculation of a virtual set of trips for the calibration of aggregated traffic models. In: $100^{\text {th }}$ Annual Meeting Transportation Research Board. Washington DC, USA.

Batista, S. F. A., Leclercq, L., 2019. Regional dynamic traffic assignment framework for mfd multi-regions models. Transportation Science 53, $1563-1590$ URL https://dx.doi.org/10.1287/trsc.2019.0921

Batista, S. F. A., Leclercq, L., Geroliminis, N., 2019. Estimation of regional trip length distributions for the calibration of the aggregated network traffic models. Transportation Research Part B: Methodological 122, 192-217.

URL https: //dx.doi.org/10.1016/j.trb.2019.02.009

Batista, S. F. A., Seppecher, M., Leclercq, L., in press. Identification and characterizing of the prevailing paths on a urban network for mfd-based applications. Transportation Research Part C: Emerging technologies.

URL https://dx.doi.org/10.1016/j.trc.2020.102953

Daganzo, C., 2007. Urban gridlock: Macroscopic modeling and mitigation approaches. Transportation Research Part B: Methodological 41, 49-62. URL https : //dx.doi.org/10.1016/j.trb.2006.03.001

Geroliminis, N., Daganzo, C., 2008. Existence of urban-scale macroscopic fundamental diagrams: Some experimental findings. Transportation Research Part B: Methodological 42, 759-770. URL https : //dx.doi.org/10.1016/j.trb.2008.02.002

Geroliminis, N., Haddad, J., Ramezani, M., 2013. Optimal perimeter control for two urban regions with macroscopic fundamental diagrams: a model predictive approach. IEEE Transactions on Intelligent Transportation Systems 14, 348-359. URL https : //dx.doi.org/10.1109/TITS.2012.2216877

Godfrey, J. W., 1969. The mechanism of a road network. Traffic Engineering and Control 11, 323-327. URL https : //trid.trb.org/view . aspx?id=117139

Haddad, J., 2017. Optimal perimeter control synthesis for two urban regions with aggregate boundary queue dynamics. Transportation Research Part B: Methodological 96, 1-25.

URL https://dx.doi.org/10.1016/j.trb.2016.10.016

Haitao, H., Yang, K., Liang, H., Menendez, M., Guler, S. I., 2019. Providing public transport priority in the perimeter of urban networks: A bimodal strategy. Transportation Research Part C: Emerging Technologies 107, 171 - 192. URL https : //dx.doi.org/10.1016/j.trc.2019.08.004

Ingole, D., Mariotte, G., Leclercq, L., 2020. Perimeter gating control and citywide dynamic user equilibrium: A macroscopic modeling framework. Transportation Research Part C: Emerging Technologies 111, $22-49$. URL https://dx.doi.org/10.1016/j.trc.2019.11.016

Jamshidnejad, A., Papamichail, I., Papageorgiou, M., De Schutter, B., 2017. Sustainable model-predictive control in urban traffic networks: Efficient solution based on general smoothening methods. IEEE Transactions on Control Systems Technology 26 (3), 813-827. URL https: //dx.doi.org/10.1109/TCST.2017.2699160

Jin, W.-L., 2020. Generalized bathtub model of network trip flows. Transportation Research Part B: Methodological 136,138 - 157. URL https: //doi.org/10.1016/j.trb.2020.04.002

Kouvelas, A., Saeedmanesh, M., Geroliminis, N., 2017. Enhancing model-based feedback perimeter control with data-driven online adaptive optimization. Transportation Research Part B: Methodological 96, 26-45.

URL https://dx.doi.org/10.1016/j.trb.2016.10.011

Lamotte, R., Geroliminis, N., 2016. The morning commute in urban areas: Insights from theory and simulation. In: Transportation Research Board $95^{\text {th }}$ Annual Meeting. pp. 16-2003.

Leclercq, L., Sénécat, A., Mariotte, G., 2017. Dynamic macroscopic simulation of on-street parking search: A trip-based approach. Transportation Research Part B: Methodological 101, 268-282.

URL https://dx.doi.org/10.1016/j.trb.2017.04.004

Lopez, C., Leclercq, L., Krishnakumari, P., Chiabaut, N., van Lint, H., 2017. Revealing the day-to-day regularity of urban congestion patterns with 3d speed maps. Scientific Reports 7, 1-11.

URL https : //dx.doi.org/10.1038/s41598-017-14237-8

Mariotte, G., Leclercq, L., 2019. Flow exchanges in multi-reservoir systems with spillbacks. Transportation Research Part B: Methodological 122, $327-349$.

URL http: //www.sciencedirect.com/science/article/pii/S019126151731175X

Mariotte, G., Leclercq, L., Laval, J. A., 2017. Macroscopic urban dynamics: Analytical and numerical comparisons of existing models. Transportation Research Part B 101, 245-267. URL https://dx.doi.org/10.1016/j.trb.2017.04.002 
Mohajerpoor, R., Saberi, M., Vu, H. L., Garoni, T. M., Ramezani, M., 2019. H $_{\infty}$ robust perimeter flow control in urban networks with partial information feedback. Transportation Research Part B: Methodological.

URL https://dx.doi.org/10.1016/j.trb.2019.03.010

Ramezani, M., Haddad, J., Geroliminis, N., 2015. Dynamics of heterogeneity in urban networks: aggregated traffic modeling and hierarchical control. Transportation Research Part B 74, 1-19.

URL https : //dx.doi.org/10.1016/j.trb.2014.12.010

Saeedmanesh, M., Geroliminis, N., 2017. Dynamic clustering and propagation of congestion in heterogeneously congested urban traffic networks. Transportation Research Procedia 23, 962-979.

URL https : //dx.doi.org/10.1016/j.trb.2017.08.021

Sbayti, H., Lu, C.-C., Mahmassani, H. S., 2007. Efficient implementation of method of successive averages in simulation-based dynamic traffic assignment models for large-scale network applications. Transportation Research Record: Journal of the Transportation Research Board 2029, 22-30.

URL https : //dx.doi .org/10.3141/2029-03

Sirmatel, I. I., Geroliminis, N., 2017. Economic model predictive control of large-scale urban road networks via perimeter control and regional route guidance. IEEE Transactions on Intelligent Transportation Systems.

URL https://dx.doi.org/10.1109/TITS.2017.2716541

Sirmatel, I. I., Geroliminis, N., 2018. Economic model predictive control of large-scale urban road networks via perimeter control and regional route guidance. IEEE Transactions on Intelligent Transportation Systems 19, 1112-1121. URL https : //dx.doi.org/10.1109/TITS.2017.2716541

Vickrey, W., 2020. Congestion in midtown manhattan in relation to marginal cost pricing. Economics of Transportation $21,100152$. URL https://dx.doi.org/10.1016/j.ecotra.2019.100152

Yang, H., Ke, J., Ye, J., 2018. A universal distribution law of network detour ratios. Transportation Research Part C: Emerging Technologies 96,22 -37 .

Yang, K., Menendez, M., Zheng, N., 2019. Heterogeneity aware urban traffic control in a connected vehicle environment: A joint framework for congestion pricing and perimeter control. Transportation Research Part C: Emerging Technologies 105, 439 - 455. URL https://dx.doi.org/10.1016/j.trc.2019.06.007

Yang, K., Zheng, N., Menendez, M., 2018. Multi-scale perimeter control approach in a connected-vehicle environment. Transportation Research Part C: Emerging Technologies 94, 32-49. URL https://dx.doi.org/10.1016/j.trc.2017.08.014

Yildirimoglu, M., Geroliminis, N., 2014. Approximating dynamic equilibrium conditions with macroscopic fundamental diagrams. Transportation Research Part B: Methodological 70, 186-200.

URL https://dx.doi.org/10.1016/j.trb.2014.09.002

Yildirimoglu, M., Ramezani, M., Geroliminis, N., 2015. Equilibrium analysis and route guidance in large-scale networks with mfd dynamics. Transportation Research Part C: Emerging Technologies 59, 404 - 420, special Issue on International Symposium on Transportation and Traffic Theory.

URL https://dx.doi.org/10.1016/j.trc.2015.05.009

Yildirimoglu, M., Sirmatel, I. I., Geroliminis, N., 2018. Hierarchical control of heterogeneous large-scale urban road networks via path assignment and regional route guidance. Transportation Research Part B: Methodological 118, 106-123.

URL https://dx.doi.org/10.1016/j.trb.2018.10.007

Zhong, R., Chen, C., Huang, Y., Sumalee, A., Lam, W., Xu, D., 2017. Robust perimeter control for two urban regions with macroscopic fundamental diagrams: A control-lyapunov function approach. Transportation Research Procedia 23, 922-941.

URL https : //dx . doi .org/10.3141/2493-09 